\title{
Interference Mitigation through Successive Cancellation in Heterogeneous Networks
}

\author{
Onur Sahin, Jialing Li, Enoch Lu, Yingxue Li, and Philip J. Pietraski \\ InterDigital Communications Inc., 4th Floor, 2 Huntington Quadrangle, South Wing, Melville, NY 11747, USA \\ Correspondence should be addressed to Onur Sahin; sahinonur@gmail.com
}

Received 30 November 2012; Accepted 2 January 2013

Academic Editors: D. Cassioli, C. Pomalaza-Ráez, and K. Teh

Copyright (C) 2013 Onur Sahin et al. This is an open access article distributed under the Creative Commons Attribution License, which permits unrestricted use, distribution, and reproduction in any medium, provided the original work is properly cited.

\begin{abstract}
We present a practical interference management scheme for heterogeneous networks (HetNets). The underlying ideas are based on (i) Han-Kobayashi-type message splitting (MS) where the receivers decode and cancel "part" of the interference which is accordingly optimized by the transmitters to ensure decoding and (ii) opportunistic interference cancellation (OIC) where the interfering transmitters act independently of the receivers that employ interference cancellation. We develop a novel transmission and reception scheme, called joint MS and OIC (MS-OIC), that engages both MS and OIC to account for a practical HetNet system with multiple macrocells and femtocells. The MS component includes a precoder design that judiciously maximizes the weighted sum throughput via the enabling of interference cancellation. A system design along with a novel scheduler that facilitates MS-OIC in a general HetNet system is also developed. System level simulations for a general HetNet system are presented, and the proposed MS-OIC scheme is compared with benchmark schemes such as Coordinated Beamforming (CBF) and joint CBF and Almost Blank Subframes (CBF-ABS). It is observed that the proposed MS-OIC scheme improves the macrocell throughput substantially, balances the achievable rates between the macrocell and femtocell users, and provides significant outage performance improvement in the system.
\end{abstract}

\section{Introduction}

The steady march of Moore's law has brought an everincreasing level of processing power not only to desktop and laptop computers but also to mobile devices. The users of these devices have come to expect broadband performance from their cellular networks to match their device capabilities, and for the most part, network capacity has grown commensurately. The resultant explosive growth in network capacity has been consistent with Cooper's Law [1] which observes that network capacity (e.g., total data delivery per month) has been doubling every 30 months since the days of Marconi and is predicted to continue for the foreseeable future. A breakdown of Cooper's law [1] reveals that the vast majority of network capacity comes from denser deployments, specifically, adding more cells. The practicality of this approach for macro deployments is now becoming questionable. Operators wish to make the most of their existing grid of macro sites and are looking towards picoand femtocells to address increased data demand, particularly in hotspot regions. The resulting mix of macro/femtocells, called a heterogeneous network (HetNet), can in principle provide cost-effective data delivery that meets the demands of the hungry user, but first there are interference issues that need to be addressed, since interference in HetNets can be substantially worse than in homogeneous macro deployments. In HetNet scenarios, it is potentially beneficial to push users into the smaller cells, since there are generally fewer users in smaller cells to compete for resources. This potentially imposes a large signal-to-interference-noise-ratio (SINR) penalty on some users sharing the same time and frequency resource. For example, the closed access femtocell has the potential to create an even larger interference problem for a macro user that happens to wander into a femtocell coverage area but is not a member of the Closed Subscriber Group (CSG) and thus cannot connect to the femto that could otherwise provide it with a large SINR signal. Instead, it forces the use of the signal from the macrocell which could have a very low SINR. A similar phenomenon happens for open access femto deployments where a macro user is associated to 
a femtocell due to range expansion $[2,3]$ and, hence, observes significant interference from the macrocell.

In this paper, we propose to utilize interference cancellation at the receivers to overcome the interference due to sharing the same time and frequency resource so that a Coordinated Multipoint (CoMP) set wide weighted sum throughput, for example, a CoMP set wide proportionally fair $(\mathrm{PF})$ weighted sum throughput, could be minimized. The interference cancellation design in this work includes the notion of a joint transceiver design along with opportunistic interference cancellation (OIC) [4] which are engaged and generalized to account for a general HetNet system and denoted as joint message splitting (MS) and OIC (denoted as MS-OIC) design. The joint transceiver design is based on the MS [5-7] concept wherein transmitted messages are split into multiple parts. Some parts (called the private parts) are transmitted at a rate that only the intended users could decode. Other parts (called the common parts) are transmitted at another rate, so that some unintended users could decode it as well as the intended user. In this way, the unintended users that can decode the common parts may subtract, or cancel, this interference using advanced receivers such as a minimum mean squared error-successive interference cancellation (MMSE-SIC) receiver, thus giving a higher effective SINR for their desired signals. On the other hand in OIC, which is also investigated in [4], the interfering transmitters do not necessarily optimize their transmission schemes to ensure interference cancellation at the unintended receivers. However, the unintended receivers try to cancel the received interference opportunistically to improve SINR.

1.1. Literature Review. The information theoretic analysis of two-user interference channel has been investigated extensively in the literature. The largest achievable region is due to Han and Kobayashi [5] which is based on the MS techniques. Recently, the extensions of the standard singleantenna model to multiple antennas have been the main effort in understanding the rate bounds of the model (see $[8,9]$ and references therein). In [8], Jorswieck and Larsson present an optimization framework for a two-user MISO system to maximize the achievable sum rate. The achievability in the paper does not consider interference cancellation techniques, and the interference is assumed as noise at the receivers. On the other hand in [9], the capacity region of the multiple-input-multiple-output (MIMO) interference channel is established for some special cases such as very strong and aligned strong interference conditions.

Similarly, the inter-cell interference problem in HetNets has drawn significant attention, and several possible solutions have been proposed in the literature. We group these proposals into four major categories as follows.

(i) Time domain solutions: victim users are scheduled in time-domain resource where the inter-cell interference is mitigated through the muting of cells, such as in Almost Blank Subframes (ABS) $[10,11]$, or time shifting of interfering signals [12]. (ii) Frequency domain solutions: inter-cell interference is mitigated through scheduling cells in reduced bandwidths to prevent overlap of (part of) signals. Some examples (such as in [12-15]) are dynamic frequency partitioning, fractional frequency reuse (FFR), and so forth.

(iii) Power setting solutions: power control [12, 13, 1618 ] is applied at aggressors to reduce interference to victim users. For example, even though reducing power in a CSG femtocell also reduces the throughput of the femtocell, it may significantly improve the performance of a macro user in the femto coverage.

(iv) Spatial domain solutions: inter-cell interference in HetNets is considered in spatial domain through coordination and/or data sharing such as in Coordinated Multipoint (CoMP) [2, 19-22]. It is also considered through interference alignment (IA) [23, 24], or interference cancellation, such as MS [25] or OIC $[4,24]$.

Among the above four categories, time domain solutions, frequency domain solutions, and power setting solutions all tend to reduce throughput in certain cells in order to aid the victim users. On the other hand, the spatial domain solutions, which generally result in superior throughput gains compared with the other solutions, may require substantial amount of information exchange among the nodes and result in much higher complexity.

CoMP-type solutions with less or no information exchange among the nodes with interference cancellation receivers are investigated in $[4,24,25]$. In [25], an MS-type transmission scheme is proposed to manage the inter-cell interference. Similar to our work in this paper, the common messages are decoded and cancelled at the neighboring cells that result in lower overall interference power. In $[4,24]$, a more practical technique, OIC, is considered where the receivers decode and cancel the interference whenever the received signal power enables such operation.

1.2. Contribution, Organization, and Notations in This Paper. Our additional contributions can be summarized as follows. We provide low-complexity precoder designs for MS to account for a practical HetNet deployment which considers the effect of inter-cell interference in a subnetwork that is composed of a set of neighboring cells, denoted as a CoMP set. The MS transmitters not only constitute their transmission schemes based on the receivers participating in MS but also consider the receivers in the neighboring cells that are affected by the resultant interference. In particular, a joint scheduler, including user equipment (UE) selection, is proposed within each CoMP set to incorporate MS and OIC techniques in a practical HetNet scenario, which forms the basis of MS-OIC scheme. The performance of the proposed MS-OIC scheme and the scheduler is presented via system level simulations. As benchmarks, the coordinated beamforming (CBF) scheme discussed in the CoMP work item [26, 27] and the joint $\mathrm{CBF}$ and $\mathrm{ABS}$ (denoted as CBF-ABS) scheme 
in enhanced inter-cell interference coordination (eICIC) work item [28] in 3GPP are considered.

Our utilization of MS to mitigate inter-cell interference in multicell environment resembles the techniques used in [25], however bearing significant differences. Initially, the system model considered in [25] is limited to single antennas at the receivers. The precoder optimization algorithm proposed in [25] is closely tied to this assumption and could not be easily generalized to the case with multiple antennas. Also in our design, the MS precoders are designed such that the interference due to the private message splits is minimized considering all victim users in the CoMP set, not only considering the pairs scheduled for MS. We achieve this by combining the MS technique with leakage-based precoder design. Moreover, our distributed interference cancellation scheme, OIC, which is not utilized in [25], is shown to provide additional gain along with the MS technique. A last point is that our MS precoder design, which uses bisection search for power optimization and closed-form based common and private precoder designs, is more efficient than the MS designs in $[25,29]$. A complexity comparison is briefly given in Section 3.1.2(d).

The system level simulations demonstrate substantial performance gains both in terms of average cell and user specific (i.e., cell-edge user) metrics (Table 4, Figures 8, 9 , and 10). Initially, it is observed that the macro users experience relatively worse effective throughputs compared with femto users where the interference can be considered as a substantial reason due to the deployment of macro users. Yet, the proposed MS-OIC scheme is shown to improve throughput fairness among macro and femto users. It is observed that MS-OIC provides around 46\% average macrocell throughput gain compared with $\mathrm{CBF}$ and indeed has a superior performance with respect to CBF-ABS in terms of all performance measures. On the other hand, MS-OIC provides an even superior performance to cell-edge users such that $360 \%$ and $39 \% 10$-percentile average macro user gains and $6 \%$ and 30\% 10-percentile average femto user gains are observed with respect to CBF and CBF-ABS, respectively. Finally, MSOIC is shown to minimize the MUE outage probability and almost mitigate it compared with CBF.

The paper is organized as follows. In Section 2, we present the system model of a multicell HetNet MIMO system and give a brief overview of the interference mitigation problem and our MS-OIC solution. The detailed designs for MS and OIC techniques, which constitute the fundamental steps in our solution, are presented in Section 3. In Section 4, a centralized scheduler which incorporates the proposed MS and OIC in a HetNet system is demonstrated, hence demonstrating our overall solution. The practical enabling mechanisms of the assumptions for the proposed schemes are given in Section 5. The isolated two-cell simulations and system level simulations are provided in Section 6, and the paper is concluded in Section 7.

Notations used in this paper are as follows. All boldface letters indicate vectors (lower case) or matrices (upper case). $\mathbf{A}^{T}, \mathbf{A}^{H}, \mathbf{A}^{-1}, \operatorname{tr}(\mathbf{A})$, and $E(\mathbf{A})$ stand for the transpose, conjugate transpose, inverse, trace, and expectation of $\mathbf{A}$, respectively. $\mathbf{I}_{a}$ signifies an identity matrix with rank $a$. For two sets $S_{a}$ and $S_{b}, \bar{S}_{a}=\left\{x \mid \forall x \notin S_{a}\right\}$ and $S_{a} \backslash S_{b}=\{x \mid x \in$ $S_{a}$ and $\left.x \notin S_{b}\right\}$.

\section{System Model, Problem Definition, and Overview of Solution}

2.1. System Model. We investigate a HetNet system which is composed of multiple macrocells. In each macrocell, multiple CSG femtocells are overlaid to the macro coverage as shown in Figure 1. All cells employ transmissions over the same time and frequency resources to fully exploit the resources in the system, and each cell has an enhanced Node B (eNB). We assume that there is at least one macro user equipment (MUE) and femto user equipment (FUE) associated with each macrocell and femtocell, respectively, where each UE is equipped with multiple antennas. Due to the closed access nature, each MUE can only be associated to a macro enhanced Node B (MeNB), but each FUE can be associated to either an MeNB or a femto enhanced Node B (FeNB). Yet in this work, we assume that each FUE is associated to an FeNB only. Moreover, each eNB schedules one UE in each transmission, and no eNB is turned "off" (or say, muted) at any time.

In order to facilitate interference management, three intrasite macrocells and the femtocells in their coverage areas form a CoMP set as shown in Figure 1. Consider one of the CoMP sets. We denote the set of eNB indices in the CoMP set as $S$ and the set of eNB indices out of the CoMP set as $\bar{S}$. In a given frequency resource, for example, a subcarrier, an eNB schedules at most one UE at a time. From the perspective of a $\mathrm{UE}$, all eNBs other than its serving eNB are interferers. If $\mathrm{UE}_{j}$ (the $j$ th $\mathrm{UE}$ ) is served by $\mathrm{eNB}_{i}$ (the $i$ th $\mathrm{eNB}$ ) and $i \in S, \mathrm{UE}_{j}$ 's received signal on the $l$ th subcarrier is

$$
\mathbf{y}_{j}^{l}=\sqrt{P_{i}^{l}} \mathbf{H}_{j i}^{l} \mathbf{W}_{i}^{l} \mathbf{x}_{i}^{l}+\sum_{k \in S \backslash\{i\}} \sqrt{P_{k}^{l}} \mathbf{H}_{j k}^{l} \mathbf{W}_{k}^{l} \mathbf{x}_{k}^{l}+\mathbf{n}_{j}^{l} .
$$

Thus, all the variables in (1) are for the $l$ th subcarrier and are defined as follows: $\mathbf{H}_{j i}^{l}$ is the $r_{j} \times t_{i}$ channel from $\mathrm{eNB}_{i}$ to $\mathrm{UE}_{j} ; r_{j}$ and $t_{i}$ are the numbers of antennas at $\mathrm{eNB}_{i}$ and $\mathrm{UE}_{j}$, respectively. Here, $P_{i}^{l}$ is the transmit power at $\mathrm{eNB}_{i}$. The $m_{i}$ codewords of $\mathrm{eNB}_{i}$ are transmitted by first mapping each one of them to one of the $m_{i}$ layers of the $m_{i} \times 1$ vector $\mathbf{x}_{i}^{l}$ (the codeword to layer mapping is assumed to be one to one). Then, $\mathbf{x}_{i}^{l}$ is precoded by the $t_{i} \times m_{i}$ normalized precoder $\mathbf{W}_{i}^{l} \cdot \mathbf{x}_{i}^{l}$ and $\mathbf{W}_{i}^{l}$ satisfy $E\left[\mathbf{x}_{i}^{l}\left(\mathbf{x}_{i}^{l}\right)^{H}\right]=\mathbf{I}_{m_{i}}$ and $\operatorname{tr}\left\{\mathbf{W}_{i}^{l}\left(\mathbf{W}_{i}^{l}\right)^{H}\right\}=$ 1 , respectively. In (1), it should be noted that the first term represents the desired signal from $\mathrm{eNB}_{i}$ to $\mathrm{UE}_{j}$; the second term represents the interference from the other eNBs in the CoMP set; the last term represents the interference from the eNBs out of the CoMP set plus additive white Gaussian noise (AWGN) with a covariance matrix

$$
\boldsymbol{\Phi}_{\text {out-set }, j}^{l}=\sum_{k \in \bar{S}} P_{k}^{l} \mathbf{H}_{j k}^{l} \mathbf{W}_{k}^{l}\left(\mathbf{H}_{j k}^{l} \mathbf{W}_{k}^{l}\right)^{H}+\sigma^{2} \mathbf{I}_{r_{j}}, \quad j \in U_{i},
$$




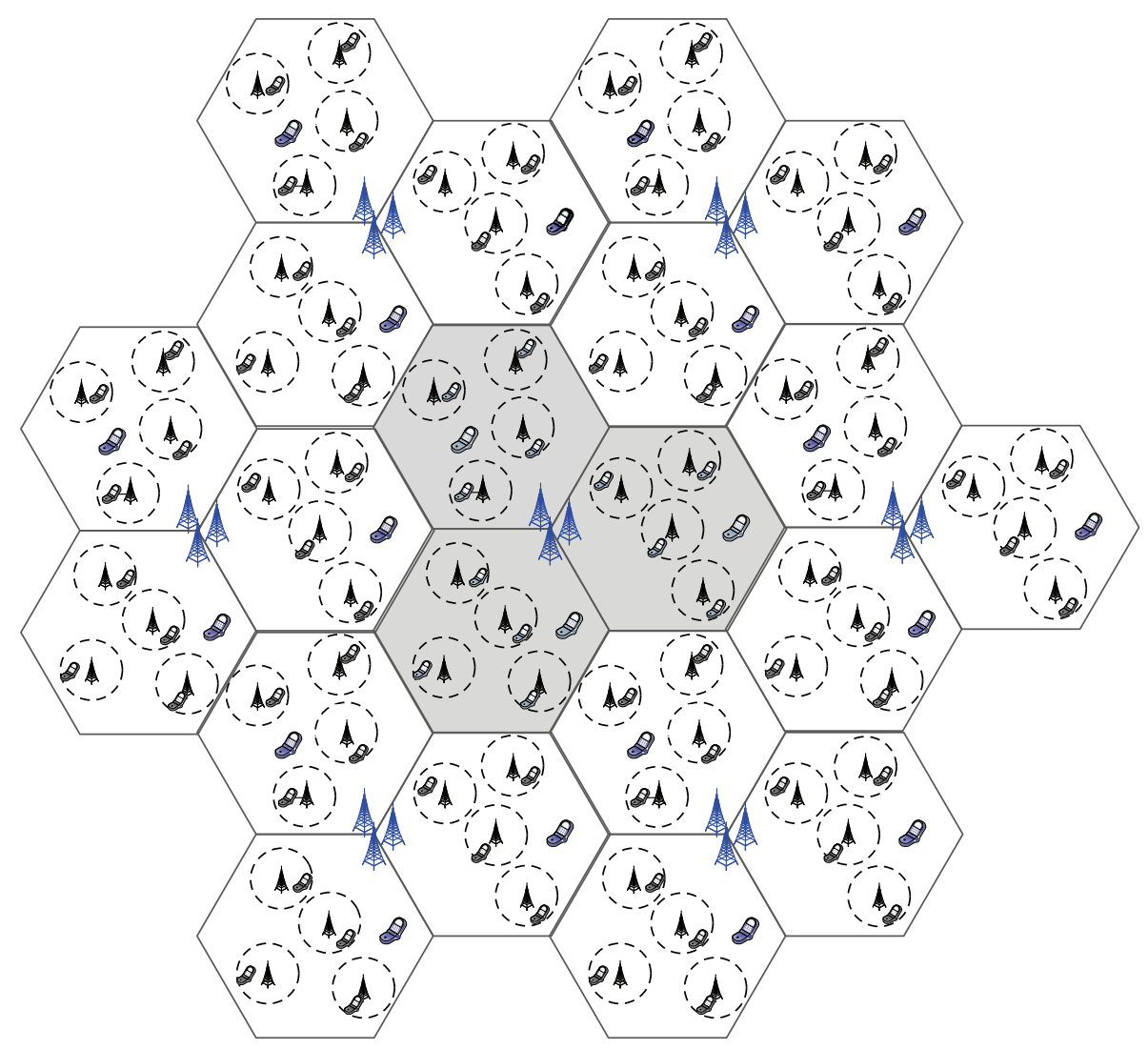

Blue: macro

Black: femto

FIGURE 1: Example of a multicell HetNet system. Shaded cells represent an example of one CoMP set. Only scheduled UEs are shown.

where $\sigma^{2}$ is the AWGN covariance. Each UE employs an MMSE-SIC receiver. Therefore, the interference from other eNBs in the CoMP set can be mitigated through coordination between eNBs within the CoMP set and/or using the UEs' interference cancellation capabilities. For example, the leakage based CBF techniques try to minimize the in-set leakage to victim UEs (subject to interference) as in $[26,27]$ (and discussed in Section 3.1.2(b)). For this purpose, we define the transmit spatial covariance matrix of the channel from $\mathrm{eNB}_{i}$ to $\mathrm{UE}_{j}$ as

$$
\mathbf{R}_{j i}^{l}=\left(\mathbf{H}_{j i}^{l}\right)^{H} \mathbf{H}_{j i}^{l} .
$$

The in-set leakage (i.e., the leakage caused to selected UEs in the other cells in the CoMP set) covariance matrix of $\mathrm{eNB}_{i}$ is then given as

$$
\mathbf{L}_{i}^{l}=\sum_{k \in S \backslash\{i\}} \sum_{j \in V_{k}} \mathbf{R}_{j k}^{l} .
$$

Here, $V_{k}$ is the set of the selected UEs indices at the $k$ th eNB. Thus, $V_{k} \subseteq U_{k}$ where $U_{k}$ is the set of the UE indices for the UEs associated to the $\mathrm{eNB}_{k}$. Note that since an FeNB has a small coverage area, it does not have to count the UEs in other macrocells in its leakage. On the other hand, an MeNB should count in its leakage the UEs in the other macrocells of its CoMP set. Since our proposed MS-OIC scheme is a spatial domain solution, for the rest of this paper, we only consider MS-OIC in one subcarrier. In light of these, for notational convenience, we drop the superscript $l$ for the $l$ th subcarrier. MS-OIC may be generalized to deal with frequency selective fading channels.

2.2. Problem Definition. The problem addressed in this paper is how to maximize the CoMP set wide weighted sum throughput by mitigating the inter-cell interference in HetNets. The dominant inter-cell interference in HetNets is the one from MeNBs to MUEs and FUEs and similarly the one from FeNBs to MUEs. The (CoMP set wide) weighted sum throughput can be defined by

$$
\max _{V_{i}, \mathbf{W}_{i}, i \in S} \sum_{i \in S} \sum_{j \in V_{i}} q_{j} g\left(\mathbf{x}_{i}, \mathbf{y}_{j}\right),
$$

where $q_{j}$ is the weight for $\mathrm{UE}_{j}$. As defined previously, $\mathbf{W}_{i}$ is the precoder at the $i$ th eNB and $V_{i}$ is the set of the selected UEs indices at the $i$ th eNB. Here, we only use a symbolic function $g\left(\mathbf{x}_{i}, \mathbf{y}_{j}\right)$ for throughput of the signal $\mathbf{x}_{i}$ received at $\mathrm{UE}_{j}$, because it is related to SINR, modulation coding scheme (MCS), and block error rate (BLER) but is not an 
analytical function. The optimization is performed through UE selection (design of $V_{i}$ ) and precoder design of scheduled UEs (design of $\left.\mathbf{W}_{i}\right)$. Since throughput $g\left(\mathbf{x}_{i}, \mathbf{y}_{j}\right)$ is not analytical, in the precoder designs in our proposed solution, we resort to deal with $R_{j i}$, the achievable rate from $\mathrm{eNB}_{i}$ to $\mathrm{UE}_{j}$, obtained by standard Shannon formula assuming i.i.d. Gaussian transmit codebooks. The MCS selection and UE selection still try to maximize the weighted sum throughput instead of the weighted sum rate.

2.3. Overview of Solution and Assumptions. Our MS-OIC solution aims at a relatively low-complexity solution. The main idea is as follows. Initially, the proposed design pairs each macrocell and one of its MUEs with an overlaid femtocell and one of its FUEs to perform MS to maximize their weighted sum throughput as well as to mitigate their interference to other victim UEs in the CoMP set. Then, in each of the unpaired femtocells, the FUE performs OIC to maximize its own throughput. Finally, the CoMP set wide weighted sum throughput could be maximized by UE selection in macrocells and femtocells, that is, MUE selection in macrocells and pairing of macrocells and femtocells in MS. In our proposed scheme, we propose the precoder designs for the scheduled UEs in the CoMP set $S$ as explained in Section 3 and specify UE selection (design of $V_{i}$ ), for example, scheduler design based on an iterative approach as given in Section 4 .

We provide a framework of our algorithm descriptions for the overall solution as follows. In Algorithm 1, we describe how the scheduling decision of the eNBs located in different macrocell is done. In Algorithm 2, we describe how MS and OIC are employed for each MeNB and its co-located femtocells (i.e., the femtocells located in the macrocell of this $\mathrm{MeNB})$.

To facilitate the corresponding precoder designs at the eNBs, we have the following assumptions of which the enabling mechanisms are discussed in Section 5. Initially, for the pair of eNBs that are scheduled to employ MS, the interpair channels (i.e., the channels from the two eNBs to the two UEs in MS), the in-set leakage covariance matrices of the two eNBs as defined in (4), and the out-of-CoMP-set interference plus noise covariance matrices for the two UEs as defined in (2) are assumed to be known at a central node (e.g., one of the eNBs of the pair), so that the transmit precoder and power optimization can be managed. Then, both eNBs in the MS pair are informed regarding the optimized precoder and power values.

Similarly, for OIC precoder design, each FeNB shares the channel state information (CSI) of its attached FUEs with the MeNB of the co-located macrocell.

Note that in our MS-OIC solution, the inter-cell interference in the CoMP set is mitigated in three different ways: firstly, some of the precoders are leakage based so as to minimize its signal leakage to victim (interfering) UEs in other cells. For example, the private precoders in MS and the OIC precoders are leakage based. Secondly, UEs perform (partial) interference cancellation in MS and OIC. In particular, the MS eNBs will jointly design the transceivers to enable partial interference cancellation at MS UEs. For details, please refer to Section 3. Lastly, when ideal channel estimation is assumed and the interfering channels are known at each UE, it is able to employ MMSE-SIC receiver that has the interference rejection capability. Through these ways, inter-cell interference from MeNBs and FeNBs in the CoMP set could be mitigated.

\section{Message Splitting and Opportunistic Interference Cancellation Algorithms}

In this section, we present our proposed MS and OIC algorithms, respectively. These algorithms are the fundamental building blocks of our overall MS-OIC solution as discussed in Section 2.3.

3.1. Message Splitting (MS). Consider two cells in Figure 2 as a pair that employs MS to manage interference, that is, an $\operatorname{MeNB}\left(\mathrm{eNB}_{1}\right)$ and its $\mathrm{MUE}\left(\mathrm{UE}_{1}\right)$, with an FeNB $\left(\mathrm{eNB}_{2}\right)$, and its FUE $\left(\mathrm{UE}_{2}\right)$. Assume that the MeNB acquires the necessary CSI information as discussed in Section 2.3 (and Section 5 in more details). In the MS design, $\mathrm{eNB}_{i}$ ( $i$ is such that $\mathrm{eNB}_{i}$ is either the MeNB or FeNB) splits its data $\mathbf{x}_{i}$ intended to its $\mathrm{UE}$ into $\mathbf{x}_{i c}$ and $\mathbf{x}_{i p}$. Here, $\mathbf{x}_{i c}$ denotes the common codewords which are aimed to be decoded at both UEs whereas $\mathbf{x}_{i p}$ denotes the private codewords to be decoded only at its UE. Upon the split of the data, $\mathrm{eNB}_{i}$ precodes $\mathbf{x}_{i c}$ and $\mathbf{x}_{i p}$ by $\mathbf{W}_{i c}$ and $\mathbf{W}_{i p}$, respectively. Finally, it sums up $\mathbf{W}_{i c} \mathbf{x}_{i c}$ and $\mathbf{W}_{i p} \mathbf{x}_{i p}$ with different weights $\sqrt{\alpha_{i c}}$ and $\sqrt{1-\alpha_{i c}}$. Since $\mathbf{W}_{i c}$ and $\mathbf{W}_{i p}$ are normalized $\left(\operatorname{tr}\left\{\mathbf{W}_{i c} \mathbf{W}_{i c}^{H}\right\}=\operatorname{tr}\left\{\mathbf{W}_{i p} \mathbf{W}_{i p}^{H}\right\}=1\right)$ and $0 \leq \alpha_{i c} \leq$ $1, \alpha_{i c}$ is used by $\mathrm{eNB}_{i}$ to allocate power to its common and private parts. Therefore, in MS, the precoder in (1) is given as

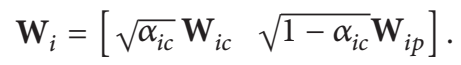

It should be noted that even though the common parts of the MeNB (resp., FeNB) do not contain data for the FUE (resp., MUE), the FUE (resp., MUE) is able to cancel it and, thus, improve the SNR of its own private codewords. The MS technique is used to achieve two goals. The first one is to maximize the weighted sum rate of these UEs, that is,

$$
\max _{\mathbf{w}_{i}, i=1,2} \sum_{i=1,2} q_{i} R_{i i}
$$

where $R_{i i}$, the achievable rate from $\mathrm{eNB}_{i}$ to $\mathrm{UE}_{j}$, is the sum of $R_{i c}$, the achievable sum rate of the common codewords, and $R_{i p}$, the achievable sum rate of the private codewords, that is,

$$
R_{i i}=R_{i c}+R_{i p}
$$

The second one is to mitigate the two eNBs' interference to other scheduled UEs in the CoMP set, which in turn helps to maximize the weighted sum rate of these UEs as well.

In the following, we demonstrate the decoding and rate expressions in Section 3.1.1 and our proposed precoder design in Section 3.1.2. 
Step 1: In iteration $l$, perform scheduling decision for $\mathrm{MeNB}_{1}$ and its co-located femtocells, assuming the other macrocells and femtocells adopt their scheduling decision in iteration $l-1$.

Step 2: In iteration $l$, perform scheduling decision for $\mathrm{MeNB}_{2}$ and its co-located femtocells, assuming $\mathrm{MeNB}_{1}$ and $\mathrm{MeNB}_{1}$ 's co-located femtocells adopt their scheduling decision in iteration $l$, and assuming $\mathrm{MeNB}_{3}$ and $\mathrm{MeNB}_{3}$ 's co-located femtocells adopt their scheduling decision in iteration $l-1$.

Step 3: In iteration $l$, perform scheduling decision for $\mathrm{MeNB}_{3}$ and its co-located femtocells, assuming the other macrocells and femtocells adopt their scheduling decision in iteration $l$.

Algorithm 1: High level scheduling of one CoMP set (having $\mathrm{MeNB}_{1}, \mathrm{MeNB}_{2}$, and $\mathrm{MeNB}_{3}$ ).

Outer loop: Search $\mathrm{MUE}_{j}$ for $\mathrm{MeNB}_{i}, i \in S$, to maximize the CoMP set wide weighted sum throughput. For each $\mathrm{MUE}_{j}$ for $\mathrm{MeNB}_{i}, i \in S$, go to the following steps.

Step 1: Pair $\left(\mathrm{MeNB}_{i}, \mathrm{MUE}_{j}, \mathrm{FeNB}_{k}, \mathrm{FUE}_{k}\right)$ to perform MS design (by Algorithm 4) to

obtain the precoders for these two eNBs.

Step 2: For each of the other femtocells located in the macrocell of $\mathrm{MeNB}_{i}$, perform

OIC design assuming $\mathrm{MeNB}_{i}, i \in S$, use the precoders designed in Step 1.

End of Steps

End of Outer loop

Algorithm 2: High Level MS-OIC scheme taking one MeNB and its co-located FeNBs as an example.

\subsubsection{Preliminaries}

Decoding. As developed in [5-7] and described briefly in Section 3.1, each UE decodes and cancels the common codewords transmitted from both eNBs, to improve the SINRs and achievable rates of its remaining private codewords. After cancelling the common codewords, each UE decodes the private codewords transmitted by its eNB by treating the private codewords from the other $\mathrm{eNB}$ as noise. As each of the two UEs treats all interference not from its paired eNB as noise, we define $\widetilde{\mathbf{n}}_{j}$ as the out-of-pair interference plus noise vector at $\mathrm{UE}_{j}(j=1,2)$. Let us also define the corresponding covariance matrix as

$$
\boldsymbol{\Phi}_{j}=E\left[\widetilde{\mathbf{n}}_{j} \widetilde{\mathbf{n}}_{j}^{H}\right]
$$

In [29], the joint decoding of the common codewords is proposed. However, this is not enabled in practical systems due to high complexity requirements. Thus, we consider a more practical sequential decoding approach which is also discussed in [29] that can be achieved via an MMSE-SIC receiver at each UE. In order to minimize the complexity further, it is assumed that the receivers decode the common codewords first, which is proceeded by private codeword decoding. Therefore, the MeNB picks a decoding order for the common codewords out of the following four cases:

(I) at $\mathrm{UE}_{1}: \mathbf{x}_{1 c}$ then $\mathbf{x}_{2 c}$, at $\mathrm{UE}_{2}: \mathbf{x}_{2 c}$ then $\mathbf{x}_{1 c}$,

(II) at $\mathrm{UE}_{1}: \mathbf{x}_{2 c}$ then $\mathbf{x}_{1 c}$, at $\mathrm{UE}_{2}: \mathbf{x}_{1 c}$ then $\mathbf{x}_{2 c}$,

(III) at $\mathrm{UE}_{1}: \mathbf{x}_{1 c}$ then $\mathbf{x}_{2 c}$, at $\mathrm{UE}_{2}: \mathbf{x}_{1 c}$ then $\mathbf{x}_{2 c}$,

(IV) at $\mathrm{UE}_{1}: \mathbf{x}_{2 c}$ then $\mathbf{x}_{1 c}$, at $\mathrm{UE}_{2}: \mathbf{x}_{2 c}$ then $\mathbf{x}_{1 c}$.
Clearly, the decoding order of the common codewords determines their achievable rates [5, 29].

After decoding and cancelling the common codewords, $\mathbf{x}_{1 c}$ and $\mathbf{x}_{2 c}, \mathrm{UE}_{1}$ and $\mathrm{UE}_{2}$ decode the private codewords, $\mathbf{x}_{1 p}$ and $\mathbf{x}_{2 p}$, respectively.

Rate Expressions. Denote $\mathrm{UE}_{1}$ and $\mathrm{UE}_{1}$, respectively, as $\mathrm{D} 1$ and $D 2$, where " $D$ " stands for destination. Also denote $S_{i c}=$ $\mathbf{W}_{i c} \mathbf{W}_{i c}^{H}, i=1,2$ as the common transmit covariance matrix and $\mathbf{S}_{i p}=\mathbf{W}_{i p} \mathbf{W}_{i p}^{H}, \quad i=1,2$ as the private transmit covariance matrix. At $\mathrm{UE}_{i}$, we define the covariance matrix of the received common codewords from $\mathrm{eNB}_{j}$ as

$$
\boldsymbol{\Omega}_{i j}^{c}=\alpha_{j c} P_{j} \mathbf{H}_{i j} \mathbf{S}_{j c} \mathbf{H}_{i j}^{H}
$$

and that of the received private codewords from $\mathrm{eNB}_{j}$ as

$$
\boldsymbol{\Omega}_{i j}^{p}=\left(1-\alpha_{j c}\right) P_{j} \mathbf{H}_{i j} \mathbf{S}_{j p} \mathbf{H}_{i j}^{H} .
$$

Therefore, at $\mathrm{UE}_{i}$, the interference covariance matrix to the common codewords is given as

$$
\mathbf{Z}_{i}=\boldsymbol{\Omega}_{i 1}^{p}+\boldsymbol{\Omega}_{i 2}^{p}+\boldsymbol{\Phi}_{i}, \quad i=1,2,
$$

where the out-of-pair interference plus noise covariance matrix $\boldsymbol{\Phi}_{i}$ is defined in (9). For each decoding order $o \in$ $\{I, I I, I I I, I V\}$ for the common codewords, the maximum rates of the common codewords $\mathbf{x}_{i c}, i=1,2$ at $D j, j=1,2$, are denoted by

$$
R_{i c, o}^{D j}=\log \left|\mathbf{I}_{r_{j}}+\alpha_{i c} P_{i} \mathbf{H}_{j i} \mathbf{S}_{i c} \mathbf{H}_{j i}^{H}\left(\mathbf{Z}_{i c, o}^{D j}\right)^{-1}\right|
$$

As can be seen in Table $1, \mathbf{Z}_{i c, o}^{D j}$ in (13) denotes the interference plus noise covariance matrix when $\mathbf{x}_{i c}$ is decoded at $\mathrm{UE}_{j}$ 


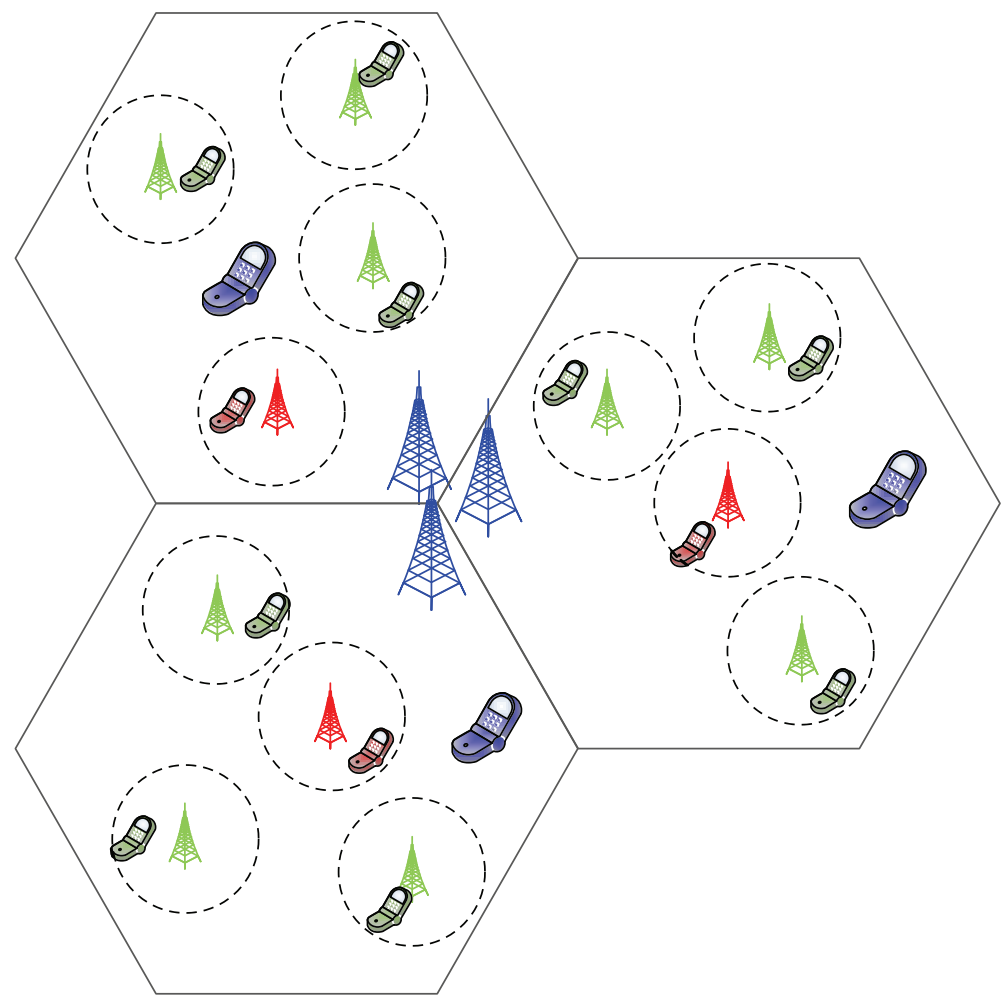

Blue and big: macro

Red: femto performing MS

Green: femto performing OIC

Figure 2: A CoMP set to illustrate our joint MS-OIC design. Only scheduled UEs are shown.

TABLE 1: Effective noise and interference covariance matrices at each UE for $\mathrm{x}_{1 c}$ and $\mathrm{x}_{2 c}$.

\begin{tabular}{lcccc}
\hline$o$ & $\mathrm{I}$ & $\mathrm{II}$ & $\mathrm{III}$ & $\mathrm{IV}$ \\
\hline $\mathbf{Z}_{1 c, o}^{D 1}$ & $\mathbf{Z}_{1}+\boldsymbol{\Omega}_{12}^{c}$ & $\mathbf{Z}_{2 c, I}^{D 1}$ & $\mathbf{Z}_{1 c, I}^{D 1}$ & $\mathbf{Z}_{1 c, I I}^{D 1}$ \\
$\mathbf{Z}_{2 c, o}^{D 1}$ & $\mathbf{Z}_{1}$ & $\mathbf{Z}_{1}+\boldsymbol{\Omega}_{11}^{c}$ & $\mathbf{Z}_{2 c, I}^{D 1}$ & $\mathbf{Z}_{2 c, I I}^{D 1}$ \\
$\mathbf{Z}_{1 c, o}^{D 2}$ & $\mathbf{Z}_{2}$ & $\mathbf{Z}_{2}+\boldsymbol{\Omega}_{22}^{c}$ & $\mathbf{Z}_{1 c, I I}^{D 2}$ & $\mathbf{Z}_{1 c, I}^{D 2}$ \\
$\mathbf{Z}_{2 c, o}^{D 2}$ & $\mathbf{Z}_{2}+\boldsymbol{\Omega}_{21}^{c}$ & $\mathbf{Z}_{1 c, I}^{D 2}$ & $\mathbf{Z}_{2 c, I I}^{D 2}$ & $\mathbf{Z}_{2 c, I}^{D 2}$ \\
\hline
\end{tabular}

according to decoding order $o, o \in\{$ I, II, III, IV $\} . \mathbf{Z}_{i c, o}^{D j}$ is expressed in terms of $\boldsymbol{\Omega}_{i j}^{c}, \boldsymbol{\Omega}_{i j}^{p}$, and $\mathbf{Z}_{i}$.

Since the common codewords are to be decodable at both UEs, the achievable rates for $o \in\{$ I, II, III, IV $\}$ are given by

$$
R_{1 c, o}=\min \left\{R_{1 c, o}^{D 1}, R_{1 c, o}^{D 2}\right\}, R_{2 c, o}=\min \left\{R_{2 c, o}^{D 1}, R_{2 c, o}^{D 2}\right\} .
$$

The achievable sum rate of the private codewords, $\mathbf{x}_{i p}$, at $\mathrm{UE}_{i}$ is given by

$$
\begin{aligned}
R_{i p}=\log \mid \mathbf{I}_{r_{i}} & \left(1-\alpha_{i c}\right) P_{i} \mathbf{H}_{i i} \mathbf{S}_{i p} \mathbf{H}_{i i}^{H} \\
& \times\left(\boldsymbol{\Phi}_{i}+\left(1-\alpha_{j c}\right) P_{j} \mathbf{H}_{i j} \mathbf{S}_{j p} \mathbf{H}_{i j}^{H}\right)^{-1} \mid,
\end{aligned}
$$

$i, j=1,2, i \neq=j$. Finally, the achievable weighted sum rate for $o \in\{$ I, II, III, IV $\}$ is given by

$$
R_{\text {sum }, o}=q_{1} R_{1 c, o}+q_{2} R_{2 c, o}+q_{1} R_{1 p}+q_{2} R_{2 p} .
$$

Note that the achievable sum rate is determined by the precoders $\mathbf{W}_{1}$ and $\mathbf{W}_{2}$. If decoding order $o^{*}$ is chosen, the achievable sum rate of the common codewords are

$$
R_{1 c}=R_{1 c, o^{*}}, \quad R_{2 c}=R_{2 c, o^{*}},
$$

and the achievable weighted sum rate of the two UEs is

$$
R_{\text {sum }}=q_{1} R_{1 c}+q_{2} R_{2 c}+q_{1} R_{1 p}+q_{2} R_{2 p} .
$$

For convenience, we denote $R_{\text {sum }}\left(\alpha_{1 c}, \alpha_{2 c}\right)$ as the achievable weighted sum rate when the power allocation is according to $\alpha_{1 c}$ and $\alpha_{2 c}$.

3.1.2. Precoder Design. From Section 3.1.1 and (13), (14), and (15), it is possible to see that in the precoder design of $\mathbf{W}_{1}$ and $\mathbf{W}_{2}$, the parameters $\mathbf{W}_{1 c}, \mathbf{W}_{2 c}, \mathbf{W}_{1 p}, \mathbf{W}_{2 p}, \alpha_{1 c}$, and $\alpha_{2 c}$ are needed to be optimized along with the best decoding order for the common codewords in order to achieve two goals: to maximize the achievable weighted sum rate and to mitigate the pair's interference to other scheduled UEs in the CoMP set. These variables and decisions are all naturally 
coupled, making the design and how to balance the two goals nontrivial. Therefore, we propose to use a suboptimal and efficient solution, such that the private and common precoder designs are based on closed form solutions and that the coupling of the power optimization, private precoder design, and common precoder design could be simple to avoid an iterative approach. In this section, we explain how the MeNB, which is entitled for the overall MS design, performs the precoder design. Basically, it searches over the power allocations $\alpha_{1 c}$ and $\alpha_{2 c}$ (Section 3.1.2(a)), performs the private and common precoder designs, calculates the corresponding $R_{\text {sum }}$ given in (18) for each pair of $\alpha_{1 c}$ and $\alpha_{2 c}$, and simply picks the power allocation and the associated design with the best $R_{\text {sum }}$ out of all candidates. Regarding the private and common precoder design for each power allocation, it performs a sequential optimization procedure. Firstly, the private precoders are designed (Section 3.1.2(b)). Secondly, upon obtaining the private precoders, the common precoders are designed and the decoding order for the common codewords is picked (Section 3.1.2(c)). We give a description of the MS design algorithm in Section 3.1.2(d).

(a) Power Optimization. In this subsection, we describe how the search over the power allocations is performed. The power optimization problem to maximize the sum rate $R_{\text {sum }}$ in (16) is not a convex optimization problem. So, let $\alpha_{i c} \in$ $\{0,1 / n, 2 / n, \ldots, 1\}$, where $i=1,2$ and $n$ is some positive integer. We empirically come up with this linear partition of the power. Other ways of partition, for example, in log scale, may also be used. Exhaustive search algorithms, as in [29], have the complexity of $O\left((n+1)^{2}\right)$, which can be prohibitive for a large set of power allocation candidates. In order to lower the complexity due to power optimization, we employ a bisection search in a two-dimensional array. For the same search precision, assuming $n$ is a power of two, the complexity is reduced to $O\left(\left(\log _{2} n+2\right)^{2}\right)$.

(b) Private Precoder Design. In this subsection, we describe the private precoder design for a fixed power allocation, $\alpha_{1 c}$ and $\alpha_{2 c}$. In order to strike a balance between the two goals and minimize the complexity, the MeNB chooses maximum signal-to-leakage-noise-ratio (SLNR) precoders [30] for itself and the FeNB. The ranks of both private precoders are chosen to maximize the sum rate of the private codewords.

Mathematically, the maximum SLNR precoder design for a given rank $m_{i p}=1, \ldots, t_{i}, i=1,2$ is given by

$$
\begin{aligned}
& \mathbf{W}_{i p}^{\mathrm{SLNR}, m_{i p}} \\
& =\underset{\substack{\operatorname{tr}\left(\mathbf{W}_{i p} \mathbf{W}_{i p}^{H}\right)=1,1 \\
\operatorname{rank}\left(\mathbf{W}_{i p}\right)=m_{i p}}}{\arg \max \left(\mathbf{W}_{i p}^{H}\left[\left(1-\alpha_{i c}\right) P_{i} \mathbf{L}_{i}+\operatorname{tr}\left(\boldsymbol{\Phi}_{i}\right) \mathbf{I}_{t_{i}}\right] \mathbf{W}_{i p}\right)}, \\
& \operatorname{tr}\left(\mathbf{W}_{i p}^{H} \mathbf{R}_{i i} \mathbf{W}_{i p}\right)
\end{aligned}
$$

where $\mathbf{R}_{i i}, \mathbf{L}_{i}$, and $\boldsymbol{\Phi}_{i}$, are defined in (3), (4), and (9), respectively. Here, $\operatorname{tr}\left(\Phi_{i}\right) \mathbf{I}_{t_{i}}$ is an artificial term to help balance the mitigation of leakage and that of noise, as in [30]. A proof similar to the one in [30] can show that

$$
\mathbf{W}_{i p}^{\mathrm{SLNR}, m_{i p}} \propto \mathbf{T}_{i}\left[\begin{array}{c}
\mathbf{I}_{m_{i p}} \\
\mathbf{0}_{\left(t_{i}-m_{i p}\right) \times m_{i p}}
\end{array}\right]
$$

where $\mathbf{T}_{i}$ is a $t_{i} \times t_{i}$ matrix, which satisfies the following:

$$
\begin{gathered}
\mathbf{T}_{i}^{H} \mathbf{R}_{i i} \mathbf{T}_{i}=\Lambda_{i}, \\
\mathbf{T}_{i}^{H}\left[\left(1-\alpha_{i c}\right) P_{i} \mathbf{L}_{i}+\operatorname{tr}\left(\boldsymbol{\Phi}_{i}\right) \mathbf{I}_{t_{i}}\right] \mathbf{T}_{i}=\mathbf{I}_{t_{i}} .
\end{gathered}
$$

In (21), $\Lambda_{i}$ is a $t_{i} \times t_{i}$ diagonal matrix with nonnegative entries. Since $\mathbf{T}_{i}$ can be found easily using the generalized eigenvalue decomposition, the MeNB effectively has a closedform solution for $\mathbf{W}_{i p}^{\mathrm{SLNR}, m_{i p}}$. So, the MeNB just repeatedly applies the above closed-form solution and determines the best ranks

$$
\left\{m_{1 p}^{*}, m_{2 p}^{*}\right\}=\left.\underset{m_{1 p}, m_{2 p}}{\operatorname{argmax}}\left(q_{1} R_{1 p}+q_{2} R_{2 p}\right)\right|_{\mathbf{W}_{i p}^{\mathrm{SLNR}, m_{i p}, i=1,2}} .
$$

Then, the private precoder is set as

$$
\mathbf{W}_{i p}=\mathbf{W}_{i p}^{\mathrm{SLNR}, m_{i p}^{*}}, \quad i=1,2 .
$$

(c) Common Precoder Design. In this subsection, we describe the common precoder design and the choosing of the decoding order (I, II, III, or IV) for given private precoders and power allocations. Note that designing the common precoder and choosing the best decoding order are coupled. Moreover, the problem of finding the common precoders and the best decoding order to maximize the common weighted sum rate $q_{1} R_{1 c, o}+q_{2} R_{2 c, o}$ is not a convex problem. Thus, we propose that the MeNB takes a heuristic suboptimal and low-complexity approach. It creates a candidate set of common precoders for itself and the FeNB. It, then, picks the combination of the two candidate sets and the decoding orders I, II, III, and IV which maximizes the sum rate for the common codewords. Mathematically, the MeNB sets $\left\{\mathbf{W}_{i c}^{W F, D 1}, \mathbf{W}_{i c}^{W F, D 2}\right\}$ to be the candidate set for $\mathrm{eNB}_{i}$, where $\mathbf{W}_{i c}^{W F, D k}$ is the maximum capacity precoder for the transmission from $\mathrm{eNB}_{i}$ to $\mathrm{UE}_{k}$ (when $\mathrm{eNB}_{j}, j \neq=i$, is silent), that is,

$$
\mathbf{W}_{i c}^{W F, D k}=\underset{\operatorname{tr}\left(\mathbf{W}_{i c} \mathbf{W}_{i c}^{H}\right)=1}{\operatorname{argmax}} \log \left|\mathbf{I}_{r_{k}}+\alpha_{i c} P_{i} \mathbf{H}_{k i}^{H} \mathbf{Z}_{k}^{-1} \mathbf{H}_{k i} \mathbf{W}_{i c} \mathbf{W}_{i c}^{H}\right|,
$$

$i, j, k=1,2, i \neq=j$. Since (24) has a closed form solution given by the well-known water-filling approach, the precoder candidate sets are created easily. Then, the following gives the best common precoder as well as the decoding order:

$$
\begin{aligned}
& \left\{\mathbf{W}_{i c}, o^{*}\right\} \\
& =\underset{\mathbf{W}_{i c} \in\left\{\mathbf{W}_{i c}^{W F, D 1}, \mathbf{W}_{i c}^{W F, D 2}\right\}, i=1,2, o \in\{\mathrm{I}, \mathrm{II}, \mathrm{III}, \mathrm{IV}\}}{\arg \max }\left(q_{1} R_{1 c, o}+q_{2} R_{2 c, o}\right) .
\end{aligned}
$$


(d) MS Algorithm Description. In this subsection, we describe the entire MS algorithm. Initially, with power allocation according to $\left(\alpha_{1 c}, \alpha_{2 c}\right)$, the precoder design and the achievable weighted sum rate $R_{\text {sum }}\left(\alpha_{1 c}, \alpha_{2 c}\right)$ are obtained as shown in Algorithm 3. Next, the MS algorithm using bisection search for power optimization is described in Algorithm 4.

Compared to existing MS designs, such as [25, 29], our proposed design tends to a lower overall complexity due to the following reasons. Initially, our bisection search in power optimization is more efficient than exhaustive search in power optimization (e.g., in [29]) and linear search of common-private rate splits (e.g., in [25], which optimizes the common-private rate split instead of optimizing the common-private power allocation directly). Moreover, our closed form solution based private and common precoder designs are more efficient than (various) iterative approaches (e.g., semidefinite programming (SDP) approaches in [25, 29]) in existing designs.

3.2. Opportunistic Interference Cancellation (OIC). In the previous section, we presented the MS design for a pair of cells (one MeNB, one MUE, one FeNB, and one FUE). Its extension to a larger network, however, may not be straightforward, or present the best performance/complexity tradeoff. In one example shown in Figure 2, where multiple femtocells (and UEs served by them) fall into a macro coverage zone, there are two obvious options to extend the MS scheme. In option 1, MeNB's data can be split into a private part and a single common part that can be decoded by all FUEs within the macro coverage. In option 2, the "common" part of the MeNB's data can be further divided into multiple substreams, each of which can only be decoded by one or a subset of FUEs. Both options not only introduce additional complexity and increased level of coordination but also limit the effectiveness of MS: in option 1, the common part is required to be decodable at all FUEs within the macrocell coverage; its data rate is limited by the weakest MeNB-FUE link. In option 2, each FUE is only able to decode a subset of "common" part; therefore, the SNR gain through interference cancellation is limited.

We, therefore, propose a low complexity approach that involves two levels of coordination between macrocells and femtocells. Still using the example shown in Figure 2, within each macrocell coverage, one FeNB (and, thus, its FUE) is first selected to pair with the co-located macrocell to form a MS pair and employ MS (for the details of femtocell selection, please refer to Section 4.1). A tight coordination occurs between the MS pair, for example, the corresponding eNBs and UEs. Between the macrocells and nonselected femtocells (referred to as OIC femtos), we propose a somewhat loose coordination-opportunistic interference cancellation (OIC). From the MS design, it is guaranteed that the common part transmitted from each MeNB can be decoded and cancelled by the paired FUE. However, no attempt is made to ensure that full or subset of macrocell interference (including both common and private parts) can be decoded and cancelled by the FUEs served by nonselected FeNBs (referred to as OIC UEs). Interference cancellation at any
OIC UE is, therefore, of opportunistic nature, depending on factors such as channel condition and macrocell precoder design.

We describe the OIC algorithm in Algorithm 5. Each OIC UE monitors the transmission scheme of its nearest macrocell (including its precoder design and data rate selection), determines whether or not such macrocell interference can be decoded and cancelled from its received signal under current channel condition, and reports to its own serving FeNB a proper channel quality indicator (CQI), based on which link adaption can be performed. Since CQI calculation already takes into account whether or not the macrocell interference can be canceled by the OIC UE, link adaptation at nonselected femtocells is able to effectively take advantage of interference cancellation capability at the OIC UE whenever it is applicable. For the precoder design at each OIC femto, we simply choose the precoder that maximizes point-to-point capacity between the OIC femto and the OIC UE it serves. Other options may include CBF precoder design that tries to minimize leakage power to other UEs.

\section{Joint Scheduler Design}

In this section, we describe a centralized scheduling algorithm for the multicell system described in Section 2. Moreover, this scheduling algorithm is used in the system level simulations to give a performance benchmark of our MS-OIC solution.

We assume that different CoMP sets are scheduled independently of each other and each CoMP set employs a centralized scheduler. For simplicity, it is assumed here that only one FUE is associated to each FeNB.

The scheduler for a CoMP set is given long-term information: the long-term CSI for each intra-CoMP set link. It is also given short-term information (delayed due to feedback delay): the channels of all the intra-CoMP set links and the out-of-CoMP-set interference plus noise covariance matrix for each of its UEs. Using this information, the scheduler performs two tasks: one is an infrequent task, that is, the creation of an MS partner list and the other is a frequent task, making the scheduling decisions for each transmission time interval (TTI).

4.1. MS Partner List. For each MUE, the scheduler picks an FeNB (and, thus, an FUE) to be its MS partner. For example, the scheduler used in the simulations (Section 6.2) picks the FeNB in the MUE's macrocell which has the strongest interference (in terms of long-term CSI) to the MUE. This MS partner list is used to minimize the search space and, thus, complexity in Section 4.2. As the list is based on the long-term CSI, it can be updated quite infrequently (e.g., once every $1 \mathrm{sec}$ ).

4.2. Scheduling Decisions for Each TTI. At each TTI, the scheduler schedules one UE per eNB: (a) it schedules one MUE and FUE in each macrocell to employ MS together; (b) it schedules all other FUEs in the CoMP set to employ OIC. The scheduler also decides the successive interference 
Step 1: For given $\alpha_{1 c}$ and $\alpha_{2 c}$, perform private precoder design to obtain $\mathbf{W}_{1 p}$ and $\mathbf{W}_{2 p}$. Sub-step 1: Design maximum SLNR precoders $\mathbf{W}_{i p}^{\mathrm{SLNR}, m_{\mathrm{ip}}}$ as in (19) for rank $m_{i p}=1, \ldots$, $t_{i}, i=1,2$. All $\mathbf{W}_{i p}^{\mathrm{SLNR}, m_{i p}}, m_{i p}=1, \ldots, t_{i}$, form a candidate set for $\mathbf{W}_{i p}, i=1,2$.

Sub-step 2: Obtain the best ranks $m_{1 p}^{*}$ and $m_{2 p}^{*}$ to maximize the weighted sum rate of the private codewords $q_{1} R_{1 p}+q_{2} R_{2 p}$ as in (22).

Sub-step 3: Obtain the private precoders $\mathbf{W}_{1 p}$ and $\mathbf{W}_{2 p}$ as in (23).

Step 2: For given $\alpha_{1 c}, \alpha_{2 c}, \mathbf{W}_{1 p}$, and $\mathbf{W}_{2 p}$, perform common precoder design to obtain $\mathbf{W}_{1 c}$ and $\mathbf{W}_{2 c}$.

Sub-step 1: Design $\mathbf{W}_{\mathrm{ic}}^{\mathrm{WF}, D k}$, maximum capacity precoder for the transmission from $\mathrm{eNB}_{i}$ to $\mathrm{UE}_{k}$ (when eNB ${ }_{j}$ is silent), as in (24). $\left\{\mathbf{W}_{i c}^{\mathrm{WF}, D 1}, \mathbf{W}_{i c}^{\mathrm{WF}, D 2}\right\}$ is the candidate set for $\mathbf{W}_{i c}, i=1,2$. Sub-step 2: Obtain the best pair of $\mathbf{W}_{1 c}$ and $\mathbf{W}_{2 c}$ and the best decoding order $o^{*}$ to maximize the weighted sum rate of the common codewords $q_{1} R_{1 c}+q_{2} R_{2 c}$ as in (25).

Step 3: Calculate the achievable weighted sum rate $R_{\text {sum }}\left(\alpha_{1 c}, \alpha_{2 c}\right)$ in (18) by using $\alpha_{1 c}, \alpha_{2 c}$, $\mathbf{W}_{1 p}, \mathbf{W}_{2 p}, \mathbf{W}_{1 c}$, and $\mathbf{W}_{2 c}$. Obtain the precoders $\mathbf{W}_{1}$ and $\mathbf{W}_{2}$ as in (6).

Algorithm 3: MS precoder design algorithm for given power allocation according to $\left(\alpha_{1 c}, \alpha_{2 c}\right)$.

Outer loop: Perform bisection search over $\alpha_{1 c}$ to maximize the achievable weighted sum rate $R_{\text {sum }}\left(\alpha_{1 c}, \alpha_{2 c}\right)$. For each $\alpha_{1 c}$, go to inner loop. The optimized $\alpha_{1 c}$ and the corresponding

$\alpha_{2 c \text {, for } \alpha_{1 c}}$ and precoders are the solution to the MS design.

Inner loop: For given $\alpha_{1 c_{-} \text {variable }}$, perform bisection search over $\alpha_{2 c}$ to maximize the $R_{\text {sum }}\left(\alpha_{1 c_{\text {_variable }}}, \alpha_{2 c}\right)$. For each $\alpha_{2 c}, R_{\text {sum }}\left(\alpha_{1 c_{\text {_variable }}}, \alpha_{2 c}\right)$ and the corresponding precoders

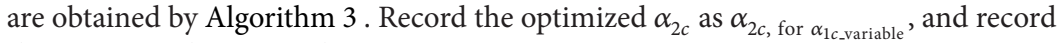
the corresponding precoders.

End of Inner loop

End of Outer loop

Algorithm 4: MS design algorithm (using bisection search).

cancellation (SIC) decoding order of the layers for each scheduled UE. Moreover, it decides the precoders, ranks, and MCSs for each eNB.

The scheduler makes all these decisions via an iterative algorithm whose goal is to maximize the sum PF throughput for the CoMP set. The algorithm cycles through the macrocells a set number of times (e.g., max iter as in Figure 3). For a macrocell, it goes through 2 steps. In the 1st step, for each MUE of this macrocell, it performs the design for the whole CoMP set under the assumption that this MUE is scheduled for MS with its MS partner. In the 2nd step, out of all the designs ( 1 for each MUE in the cell), it picks the one which maximizes the sum PF weighted throughput for the whole CoMP set. After the algorithm finishes cycling through the macrocells, it terminates. Figures 3 and 4 illustrate this iterative algorithm by denoting the macrocells of the CoMP set as macrocell 1 , macrocell 2, and macrocell 3 and by denoting the MUEs of a macrocell as MUE 1, MUE 2, and so forth (note this slight deviation from the notation of Section 2 is for presentation simplicity).

Now, let us assume the algorithm is currently up to MUE $j$ of macrocell $k$. We will devote the rest of this subsection to explain how the algorithm performs the design for the whole CoMP set under the assumption that MUE $j$ and its MS partner are considered to perform MS in macrocell $k$. Firstly, the algorithm performs the MS design for MUE $j$ and its MS partner. This is achieved by the steps as explained in Section 3.1.2(d) except that this algorithm uses the weighted (possible weights are given in Section 4.3) sum throughput for the pair instead of sum rate. Secondly, the algorithm performs the OIC design for all femtocells in macrocell $k$, except the MS partner of MUE $j$. For a given FeNB, it uses the design process in Section 3.2 except for one small difference-it uses the supportable MCS, instead of rate, to determine whether an interfering MeNB layer can be decoded or not. Lastly, the algorithm updates the MCSs and decoding orders for all currently scheduled UEs in the CoMP set. Note that for each of the OIC FUEs, this last step may change the number of interfering MeNB's layers to cancel.

4.3. Weights for the MS Design of a Pair. For convenience, call the weight for the MUE's (resp., FUEs) throughput the macro (resp., femto) weight. For fairness, one may consider the MUE's (resp., FUEs) PF weight for the macro (resp., femto) weight. However, the MeNB may serve multiple MUEs while the FeNB only serves the FUE of this pair. Thus, the PF weight for the MUE may be much larger than for its MS partner. This observation leads us to set the macro (resp., femto) weight be the PF weight for the MUE (resp., FUE) divided by the number of UEs served by the MeNB (resp., FeNB) in the simulations. That is, the weight for $\mathrm{UE}_{i}$ at TTI $n$ is $v_{i}^{(n)} / N_{i}$ 
Outer loop: The $\left(\mathrm{MeNB}_{i}, \mathrm{MUE}_{j}, \mathrm{FeNB}_{k}, \mathrm{FUE}_{k}\right)$ are paired to employ $\mathrm{MS}$ as given in

Algorithm 4 with the precoders $\mathbf{W}_{1}$ and $\mathbf{W}_{2}$. MeNB $\mathrm{B}_{i}$ transmits $m_{1}$ codewords.

Step 1: Let $c=1$.

Step 2: $\mathrm{FUE}_{t}$ of $\mathrm{FeNB}_{t}, t \neq=k$, tries to decode and cancel codeword $c$ of $\mathrm{MeNB}_{i}$

$\left(c \in\left\{1, \ldots, m_{1}\right\}\right)$, using MMSE-SIC receiver.

Sub-step 1: If codeword $c$ is decoded and cancelled successfully, for example, $R_{c}^{\mathrm{FUE}_{t}} \geq$

$R_{c}^{\mathrm{MUE}_{i}}$, optimize the precoder at $\mathrm{FeNB}_{t}\left(\mathrm{~W}_{t}\right)$ to maximize the rate of $\mathrm{FUE}_{t}$ where

$\mathrm{W}_{t}$ is given by (24) and replacing $\mathrm{Z}_{k}$ with the received interference plus noise

covariance matrix after cancellation of layers $\{1, \ldots, c\}$. Go to Step 3 .

Sub-step 2: If codeword $c$ could not be decoded correctly, for example, $R_{c}^{\mathrm{FUE}_{t}}<R_{c}^{\mathrm{MUE}_{i}}$, go to "end of Outer loop."

Step 3: $c=c+1$. If $c \leq m_{1}$, go to Step 2 .

End of Outer loop

Algorithm 5: OIC algorithm.

where $v_{i}^{(n)}$ denotes the PF weight for $\mathrm{UE}_{i}$ at TTI $n$ and $N_{i}$ denotes the number of UEs served by the serving eNB of $\mathrm{UE}_{i}$.

\section{Enabling Mechanisms}

In this section, we demonstrate the practical challenges and corresponding proposed solutions to our interference cancellation schemes presented in this paper. Specifically, we discuss the, impact of the assumptions made for our proposed approach (see Section 2.2) to the system design.

As already discussed, the MS design assumes that the interpair channels (i.e., the channels from the two eNBs to the two UEs in MS), the in-set leakage covariance matrices of the two eNBs as defined in (4), and the out-of-CoMP-set interference plus noise covariance matrices for the two UEs as defined in (2) are known at a central controller such as one of eNBs of the MS pair. This is accomplished in two steps. (a) Each of the two UEs feeds back the CSI for its links from both eNBs. Each UE that is in other cells in the CoMP set and is a potential victim affected by either of the two eNBs needs to feed back the necessary transmit spatial covariance matrix to its serving eNB. (b) Each of the potential victim UEs' serving eNB shares the CSI with the aggressor eNB(s), via X2 interface. Then, one of the eNBs shares its CSI with the other, for example, via X2 interface. Once one of the two eNBs has all the CSI, it performs the MS design and sends the necessary information to the other eNB, for example, via $X 2$ interface. Currently, there are ongoing 3GPP discussions about adding a direct $X 2$ connection and a $X 2$ through gateway connection between MeNB and FeNB [31, 32]. The main reason is to aid mobility and handoffs between macros and femtos. The main hurdles, on the other hand, seem to be concerned about the reliability and whether such a connection is necessary [33]. For example, since the broadband used to backhaul residential FeNBs does not have any quality of service (QoS) guarantee, there would, thus, also be no guarantee for the $X 2$ connections using it. Nevertheless, such a connection could be easily used to share CSI, precoders, modulation and coding schemes (MCSs), and so forth between an MeNB and FeNB in its macrocell as needed above.

In OIC, an FUE first determines if the interference from the nearest MeNB can be cancelled. Then, it feeds back the CQI accordingly to its FeNB, which in turn determines its own transmission schemes (precoder, MCSs, etc.). Note that the MeNB does not intend to aid the OIC FUEs cancellations in designing its transmission. However, the OIC FUE needs to know the characteristics of the MeNB interference ahead of time (before the interference is transmitted over the air), so that it can correctly calculate CQI when the interference is actually transmitted. In practice, MeNB can broadcast the transmission parameters (precoder, MCSs) ahead of time over a special channel which can be monitored by all FUEs within its coverage.

The second set of assumptions shares some similarities with the first. Each FeNB shares its CSI with the MeNB of the co-located macrocell via an X2 connection. Since the MeNBs of a CoMP set are collocated, this means that a centralized entity has CSI for the CoMP set (whatever the FeNBs send to the MeNBs). Consequently, a centralized scheduler for each CoMP set (as in Section 4) does the MS designs and OIC decision making for the CoMP set.

\section{Simulation Results}

This simulation results section has two parts. In Section 6.1, our MS algorithm is compared with the baselines from the literature in a link-level simulation. On the other hand, in Section 6.2, we test our MS-OIC solution in a system level simulation. A CoMP baseline and eICIC baseline are also simulated to compare the performances of the three state-of-the-art solutions in mitigating inter-cell interference in HetNets. We use $n=4$ in power optimization in Section 3.1.2(a). As we have seen in the simulation results, $n=$ 4 is sufficient to achieve decent performance with relatively low complexity. Our other results (not presented here) show that further increasing $n$ only leads to very small performance improvement. 


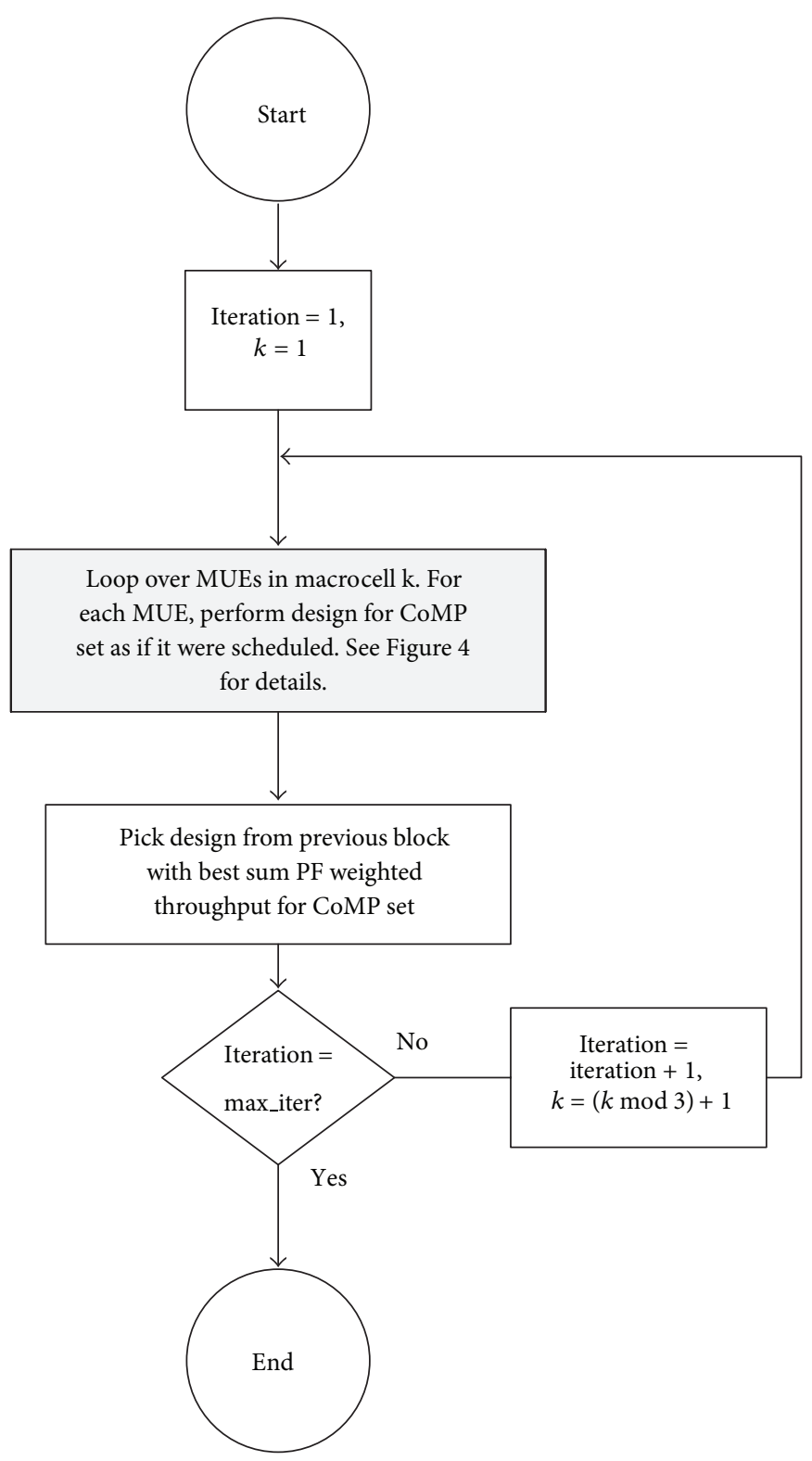

FIgURE 3: Flow chart for the scheduler of a CoMP set.

All simulation results are obtained using MATLAB.

6.1. Isolated Two-Cell Simulations. In this subsection, we consider an isolated two-cell system with the channel model given below, which is also investigated in Section IV of [29],

$$
\begin{aligned}
& \mathbf{y}_{1}=\mathbf{H}_{11} \mathbf{W}_{1} \mathbf{x}_{1}+a \mathbf{H}_{12} \mathbf{W}_{2} \mathbf{x}_{2}+\widetilde{\mathbf{n}}_{1}, \\
& \mathbf{y}_{2}=a \mathbf{H}_{21} \mathbf{W}_{1} \mathbf{x}_{1}+\mathbf{H}_{22} \mathbf{W}_{2} \mathbf{x}_{2}+\widetilde{\mathbf{n}}_{2} .
\end{aligned}
$$

Let all the eNBs and UEs have the same number of antennas. The channel model in (1) is amended by introducing one scalar parameter $a$ (which allows easy control of the average interference power) and by letting the out-of-pair interference plus noise covariance matrix $\boldsymbol{\Phi}_{i}=\mathbf{I}_{r_{i}}$ for $i=$ 1,2. For comparison, we consider the following baseline schemes: (i) "interference free transmission" assuming no inter-cell interference, an ideal upper bound of all schemes; (ii) time division multiplexing (TDM) in [29, Section III.C], an upper bound scheme of the LTE Release 10 baseline scheme ABS; (iii) "Shang et al.," our implementation of [29]; (iv) "MS (exhaustive search)," using our proposed private and common precoder designs but exhaustive search for the power allocation. On the other hand, our proposed MS technique is denoted as "MS (bisection search)" only in this subsection to differentiate from "MS (exhaustive search)."

In Figure 5, we compare all schemes' achievable sum rates as the number of antennas for each node increases, when $a=$ $1 / \sqrt{3}$ as in [29]. "MS (bisection search)" outperforms "TDM," which implies that it also outperforms ABS. The bisection search for power allocation provides a good performance and complexity tradeoff, compared to exhaustive search. Moreover, "MS (bisection search)" performs very close to 


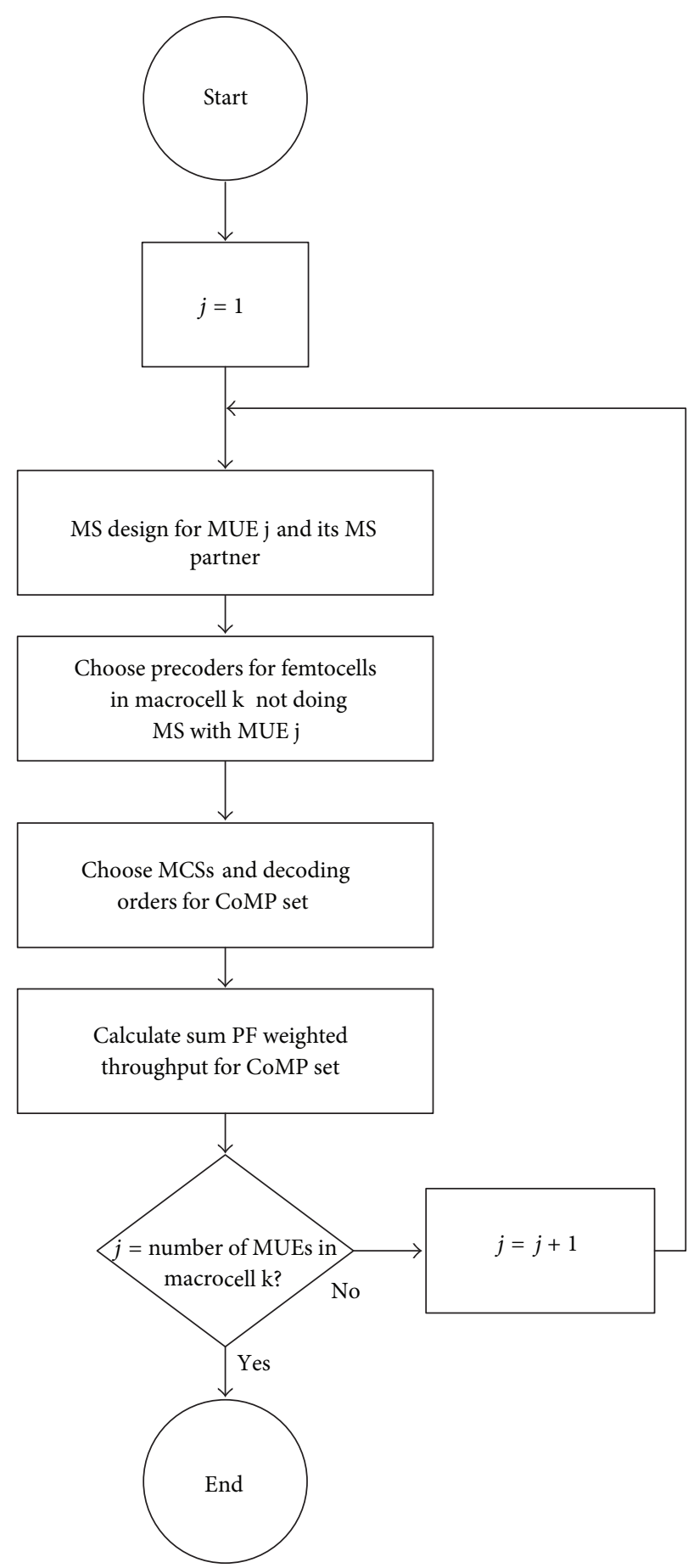

FIgURE 4: Flow chart for shaded block in Figure 3.

"Shang et al." [29] which uses the ideal joint decoding and exhaustive search over power allocations, with only $0.44 \%$ sum rate degradation.

On the other hand, in Figure 6, each node has 8 antennas and we evaluate the achievable sum rate as a function of average performance power, defined as $10 \log _{10} a^{2} \mathrm{~dB}$ as in [29]. Again, we observe that "MS (bisection search)" outperforms "TDM" and performs very close to "MS (exhaustive search)." Moreover, the sum rate of "MS (bisection search)" converges to those of "Shang et al." [29] and "interference free transmission" in strong interference region. As interference becomes weaker, the curve of "MS (bisection search)" is further away from those of "Shang et al." [29] and "interference free transmission." In the weak interference region, unlike "Shang et al." [29], whose sum rate converges to that of "interference free transmission," "MS (bisection search)" 


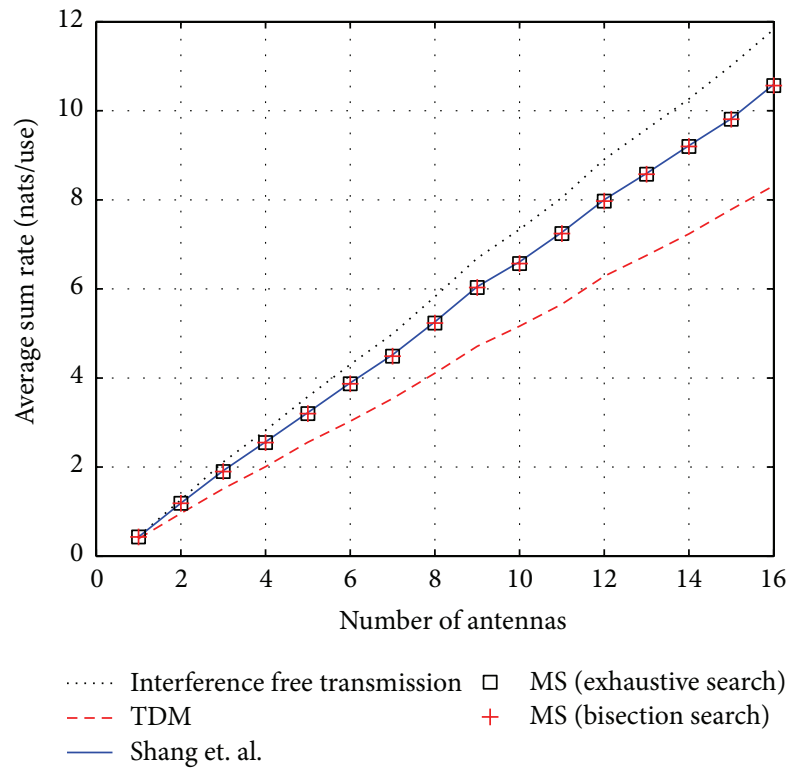

FIGURE 5: Average achievable sum rate as a function of number of antennas.

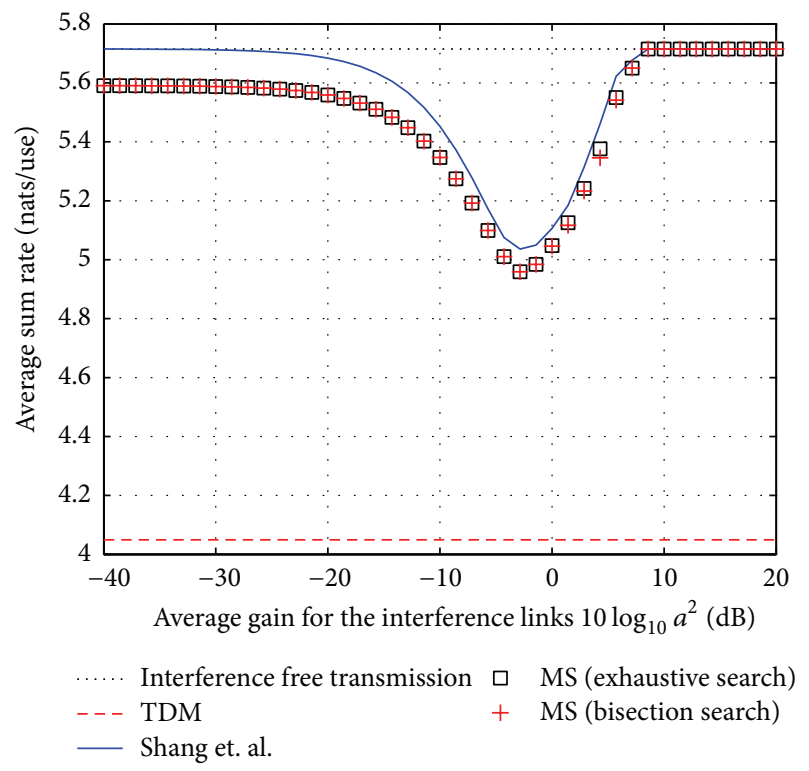

Figure 6: Achievable sum rate as a function of average interference power for 8 antennas at both eNBs and UEs.

has $\sim 2.2 \%$ sum rate degradation. This is mainly due to the fact that "MS (bisection search)" tends to transmit only the private parts in this region and the max SLNR based private precoder design is strictly suboptimal in maximizing the sum rate.

\subsection{System Level Simulations}

6.2.1. System Assumptions. In the system level simulations, we consider a special case of the multicell system model described in Section 2. In this case, there are 21 macrocells

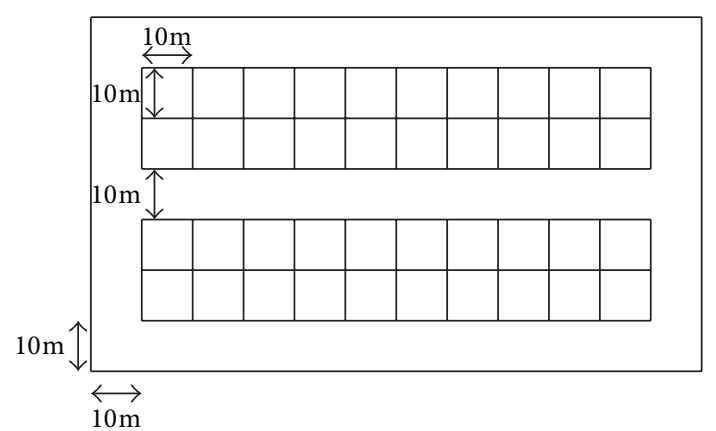

FIGURE 7: Floor plan of one floor in a femto block in the dual stripe model.

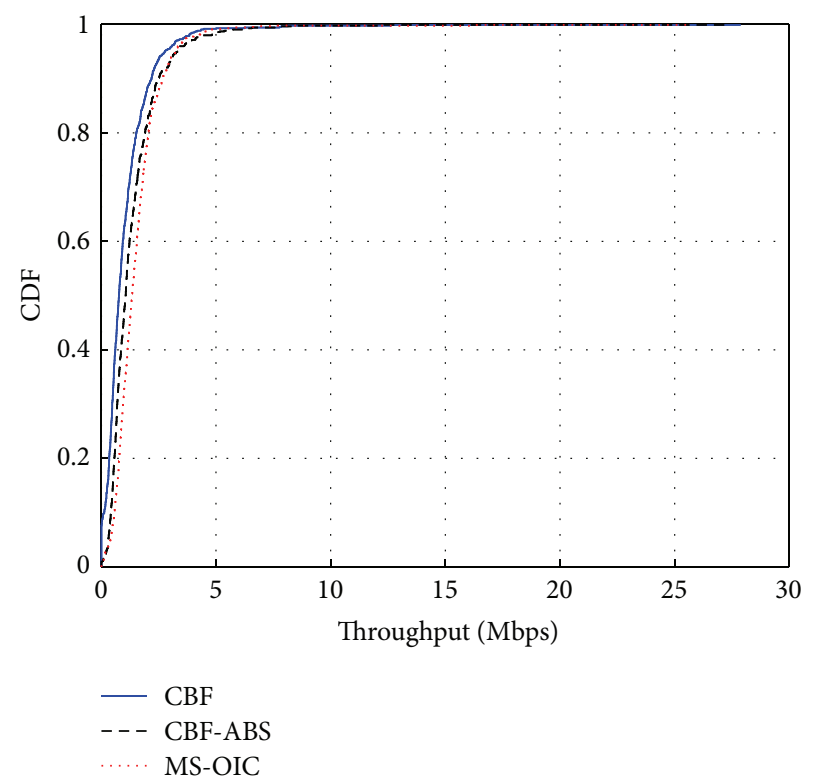

FIGURE 8: CDF of MUE throughputs.

and 4 femtocells overlaid in each macrocell. The macrocells are deployed as in the standard LTE-A deployment [2] with inter-site distance of $500 \mathrm{~m}$. Each cell site is at the intersection of three adjacent hexagonal macrocells. The femtocells are deployed as in the dual stripe model [2]. In each macrocell, one femto block (building) with 4 floors is dropped. On each floor, there are hallways and 40 rooms, each being $10 \mathrm{~m} \times 10 \mathrm{~m}$ (see Figure 7). An FeNB is randomly dropped in a random room on each floor. Three cosited MeNBs and the 12 FeNBs in their cells form one CoMP set.

In each macrocell, 10 MUEs are randomly dropped, 6 inside the macrocell's femto block and 4 outside. In each femtocell, there is 1 FUE (dropped randomly in the same room as the FeNB). Since all FUEs are indoors, this means approximately $70 \%$ of UEs are indoors. The percentage $70 \%$ is chosen, since it is observed that $70-80$ percent of mobile traffic is generated indoors, mostly in the home or office [34]. The UEs are associated to the eNBs using the Biased-RSRP method [2] in LTE with $25 \mathrm{~dB}$ biasing for FeNBs. The biasing is used to ensure each FUE is served by one FeNB. 
TABLE 2: ABS pattern. $(*)$ indicates muted.

\begin{tabular}{|c|c|c|c|c|c|c|c|c|c|c|}
\hline & TTI 1 & TTI 2 & TTI 3 & TTI 4 & TTI 5 & TTI 6 & TTI 7 & TTI 8 & TTI 9 & TTI 10 \\
\hline \multicolumn{11}{|c|}{ Macrocell } \\
\hline Femto 1 & * & * & & & & & & & & \\
\hline Femto 2 & & & * & * & & & & & & \\
\hline Femto 3 & & & & & * & * & & & & \\
\hline Femto 4 & & & & & & & $*$ & $*$ & & \\
\hline
\end{tabular}

TABLE 3: Throughputs and MS-OIC's throughput gains.

\begin{tabular}{lccccc}
\hline & CBF & CBF-ABS & MS-OIC & MS-OIC gain over CBF (\%) & MS-OIC gain over CBF-ABS (\%) \\
\hline Avg. cell throughput (Mbps) & 51.62 & 42.76 & 51.40 & -0.42 & 20.20 \\
Avg. macrocell throughput (Mbps) & 10.56 & 13.60 & 15.41 & 45.90 & 13.33 \\
Avg. femtocell throughput (Mbps) & 61.89 & 50.06 & 60.40 & -2.40 & 20.67 \\
10th percentile avg. MUE throughput (Mbps) & 0.12 & 0.41 & 0.57 & 362.75 & 38.92 \\
10th percentile avg. FUE throughput (Mbps) & 45.73 & 37.31 & 48.60 & 6.28 & 30.26 \\
\hline
\end{tabular}

TABLE 4: MUE outage probabilities.

\begin{tabular}{lccc}
\hline & CBF & CBF-ABS & MS-OIC \\
\hline MUE outage probability & 6.79 & 0.95 & 0.48 \\
\hline
\end{tabular}

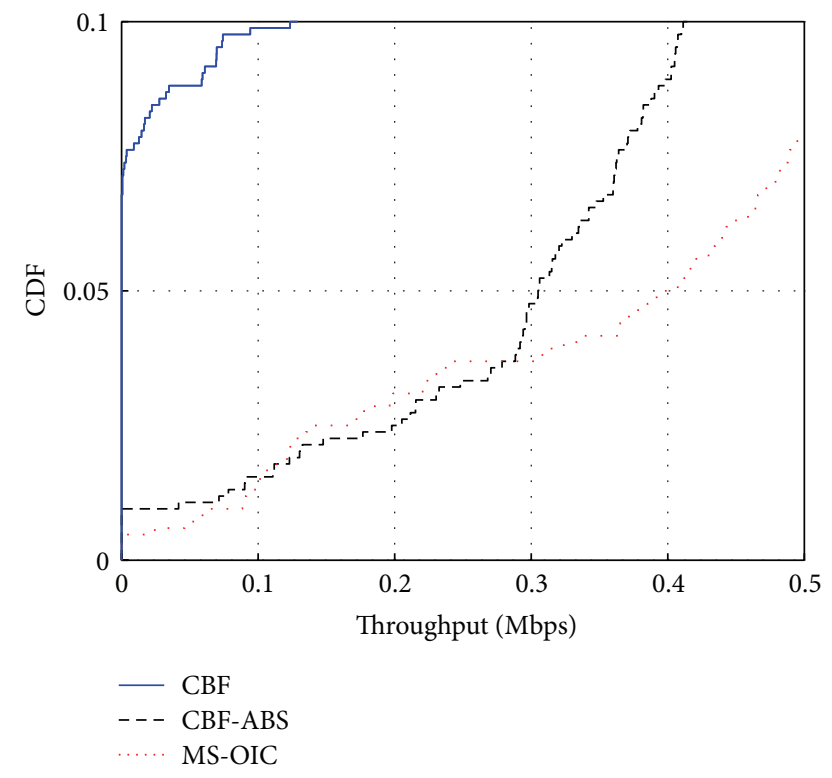

FIGURE 9: Zoomed in CDF of MUE throughputs.

We assume a frequency flat fading channel model. The transmit powers at each MeNB and FeNB are $46 \mathrm{dBm}$ and $30 \mathrm{dBm}$, respectively. The antenna gains at each MeNB and FeNB are $14 \mathrm{dBi}$ and $5 \mathrm{dBi}$, respectively. And the noise figure at each $\mathrm{UE}$ is $7 \mathrm{~dB}$. Penetration loss is accounted for indoor UEs. A full buffer traffic model is assumed. The wrap around technique is used to avoid edge effects due to the finite map size. Lastly, simulations are run for 4 drops, each drop having duration of 2000 TTIs.

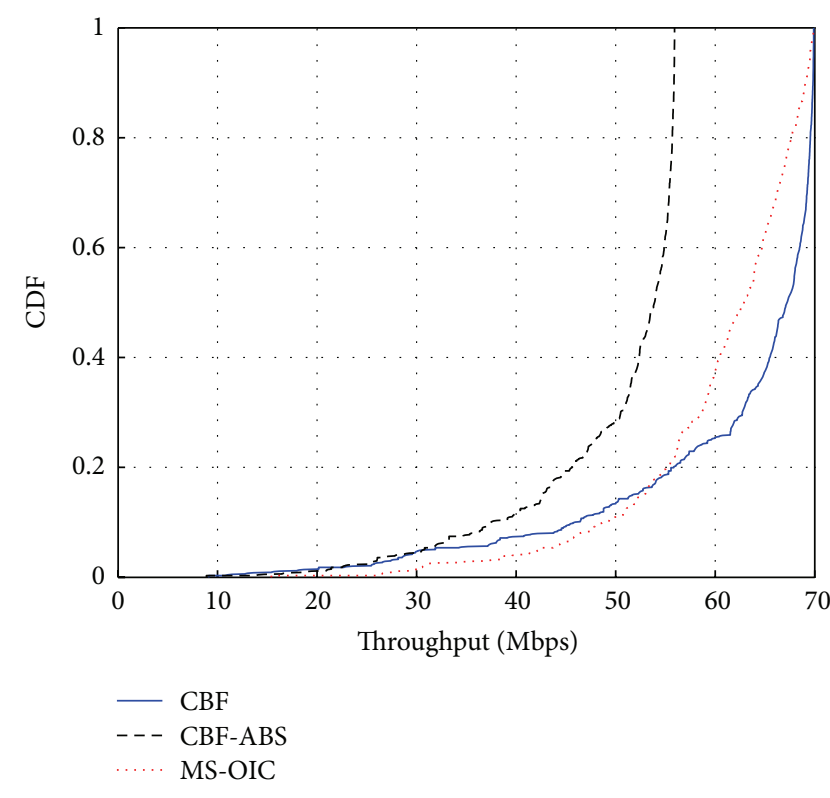

Figure 10: CDF of FUE throughputs.

6.2.2. Investigated Schemes. MS-OIC and 2 baselines, CBF and CBF-ABS, are investigated. All schemes seek to make a good tradeoff between maximizing the average performance and fairness. To do so, they all employ PF weights. There are multiple ways to define PF weights. The one definition used here is the same as the one in the generic PF scheduler for OFDMA in IEEE $802.16 \mathrm{~m}$ [35]. That is, the PF weight of $\mathrm{UE}_{i}$ in the $n$th TTI is

$$
v_{i}^{(n)}=\frac{1}{\left(\bar{T}_{i}^{(n-1)}\right)^{\beta}} .
$$


Here, $\bar{T}_{i}^{(n)}$ is the exponentially weighted moving average (EWMA) throughput (in Mbps) of $\mathrm{UE}_{i}$ from the 1st to the $n$th TTI, that is,

$$
\bar{T}_{i}^{(n)}=(1-\gamma) \bar{T}_{i}^{(n-1)}+\gamma T_{i}^{(n)}, \quad n=1,2, \ldots,
$$

where $T_{i}^{(n)}$ is the instantaneous throughput (in Mbps) of $\mathrm{UE}_{i}$ in the $n$th TTI with initialization $\bar{T}_{i}^{(0)}=0.001 \mathrm{Mbps} ; \gamma=$ 0.0029 is the decaying factor and $\beta=1.4$.

More details for each of the 3 schemes are as follows.

CBF. In CBF, each CoMP set has a centralized scheduler, which is independent from the schedulers of the other CoMP sets. The scheduler for a CoMP set has the out-of-CoMP-set interference plus noise power for each UE. It also has the transmit spatial covariance matrices (see (3) for definition) from each eNB in the CoMP set to each UE in the CoMP set. The scheduler performs UE selection to maximize the PF weighted sum capacity for the CoMP set. For the precoder design, it performs the max SLNR precoder design with rank adaptation for each eNB. The metric for the rank selection is the single cell throughput for the eNB. The MCS is chosen such that the target BLER is 0.1. The UEs use minimum mean square error (MMSE) receivers without SIC.

$C B F-A B S$. This is the same as the CBF scheme except for one difference. Namely, the femtocells in any given macrocell, label them femtos 1-4, are muted according to the pattern in Table 2 . The pattern repeats every 10 TTIs. Note that ABS are an LTE Release 10 baseline scheme. An ABS pattern is not necessarily an optimal TDM mode in maximizing the system performance.

MS-OIC. The scheduler and CSI requirements are precisely those given in Section 4. The specific MS and OIC designs used in the scheduler are as described in Section 3. The MCS is chosen such that the target BLER for the common and private codewords are 0.01 and 0.1 , respectively. For all nonMS codewords, the target BLER is 0.1. It is assumed that the common parts at both UEs in MS can always be decoded correctly because of the low target BLER.

6.2.3. Simulation Results. The throughputs (in Mbps) of MSOIC and the two baselines are given in Table 3. The gains (in \%) of MS-OIC over each baseline for the throughput metrics are also conveniently given in the same table. Note that the average cell throughput in the above-mentioned table is actually equal to the average macrocell throughput times $1 / 5$ plus the average femtocell throughput times $4 / 5$. This is simply because there is four times the amount of femtocells as macrocells. The MUE outage probabilities are given in Table 4. The CDF plot of the MUE throughputs is given in Figure 8 and zoomed in plot to show the lower percentile results in Figure 9. The CDF plot of the FUE throughputs is given in Figure 10. Both MS-OIC and CBF-ABS hope to improve in how CBF deals with the MUE dead zone problem. So, we shall first compare MS-OIC and CBF. Then finally, we compare the effectiveness, if any, of MS-OIC and CBF-ABS.
We observe in Table 3 that for the deployment explained in Section 6.2.1, compared to CBF, MS-OIC provides $46 \%$ gain in average macrocell throughput while having $0.5 \%$ degradation in average cell throughput which is due to $2.4 \%$ loss in average femtocell throughput. Considering the relatively high throughput of femtocell, we argue that MS-OIC suffers an insignificant loss in average femtocell throughput to provide a very large and needed gain in average macrocell throughput. The gains are even more impressive in the cell edge (see MUE outage probability drops from $6.79 \%$ to $0.48 \%$ in Table 4 and 10th percentile throughput gain of $363 \%$ for MUEs and $6 \%$ for FUEs in Table 3). The 10th percentile throughput and outage probabilities can also be seen in the CDF plots, in Figures 8, 9, and 10. The MUE throughput CDF plots also show that the curve for MS-OIC is almost always to the right of the CBF one. So, MS-OIC helps almost all but some of the highest throughput MUEs. Looking at the FUE throughput CDF plots, one can see MS-OIC does not benefit only the 10th percentile FUE throughput. It actually benefits all FUEs up to around the 15th percentile. In summary, MS-OIC significantly helps the MUEs-especially the disadvantaged ones-without significantly degrading the femto throughputs. Moreover, it actually benefits the lower percentile FUE throughputs.

The last comparison is MS-OIC versus CBF-ABS. Considering Tables 3 and 4, it is easy to see that MS-OIC provides significant gains for all metrics in the tables, both for the macro and femto. From the MUE throughput CDF plots, it is also easy to see that MS-OIC provides better throughputs for most percentiles. For the FUE throughput CDF plots, it is even more obvious. By muting the femtos, CBF-ABS does provide macrocell gain over $\mathrm{CBF}$. However, the gain is not nearly as much as MS-OIC's, and CBF-ABS severely hurts the femto performance with an average cell throughput degradation from $61.89 \mathrm{Mbps}$ to $50.06 \mathrm{Mbps}$. From analyzing the results, it is clear that the performance of MS-OIC is much more desirable than that of CBF and CBF-ABS.

\section{Conclusion}

In this work, we present a novel interference management scheme in HetNets considering the practicality constraints in the implementation in the systems. Our ideas are based on the interference cancellation at the destinations using MMSESIC type receivers. Our proposed interference management scheme is based on the MS technique of Han-Kobayashi [3] and OIC. It is, thus, called joint MS and OIC (MS-OIC). In the general HetNet setup, our MS-OIC targets employing MS in macrocell and femtocell pairs, whereas at the same time reducing the interference to some neighboring cells. Moreover, the remaining femto users try to opportunistically cancel the received interference. We provide an overall system design framework where the receivers are implemented with interference cancellation capabilities.

Our system level simulation results demonstrate that our proposed MS-OIC provides around 20\% average cell throughput gain compared with $3 \mathrm{GPP}$ benchmark model 
CBF-ABS where both macro and femto users enjoy throughput improvements. On the other hand, considering another 3GPP benchmark model CBF, it is shown that average macrocell throughput, which is usually degraded due to the presence of small cells in its coverage, is shown to increase substantially with respect to another benchmark model of 3GPP with slight degradation at the average femtocell throughput. Hence, the proposed interference cancellation scheme MS-OIC further imposes fairness between macrocells and femtocells in HetNets.

\section{Acknowledgments}

The authors would like to thank Dr. Xiaohu Shang for his help in their understanding of the implementation of algorithms in [29]. The authors also would like to thank the editors for their constructive comments that helped to improve the paper.

\section{References}

[1] M. Cooper, "Cooper's Law," Arraycomm, http://www.arraycomm.com/technology/coopers-law.

[2] 3GPP TR 36.814 v9.0.0, "Further advancements for E-UTRA physical layer aspects (Release 9)," March 2010.

[3] D. Lopez-Perez, X. Chu, and I. Guvenc, "On the expanded region of picocells in heterogeneous networks," IEEE Journal of Selected Topics in Signal Processing, vol. 6, no. 3, pp. 281-294, 2012.

[4] S. Rangan, "Femto-macro cellular interference control with subband scheduling and interference cancelation," in Proceedings of IEEE Globecom Worshop on Femtocell Networks, December 2010.

[5] T. S. Han and K. Kobayashi, "A new achievable rate region for the interference channel," IEEE Transactions on Information Theory, vol. 27, no. 1, pp. 49-60, 1981.

[6] A. B. Carleial, "Interference channels," IEEE Transactions on Information Theory, vol. 24, no. 1, pp. 60-70, 1978.

[7] C. Suh and D. N. C. Tse, "Feedback capacity of the gaussian interference channel to within 2 bits," IEEE Transactions on Information Theory, vol. 57, no. 5, pp. 2667-2685, 2011.

[8] E. A. Jorswieck and E. G. Larsson, "Monotonic optimization framework for the two-user MISO interference channel," IEEE Transactions on Communications, vol. 58, no. 7, pp. 2159-2168, 2010.

[9] X. Shang, B. Chen, G. Kramer, and H. V. Poor, "Capacity regions and sum-rate capacities of vector Gaussian interference channels," IEEE Transactions on Information Theory, vol. 56, no. 10, pp. 5030-5044, 2010.

[10] D. López-Pérez, I. Güvenç, G. D. L. Roche, M. Kountouris, T. Q. S. Quek, and J. Zhang, "Enhanced intercell interference coordination challenges in heterogeneous networks," IEEE Wireless Communications, vol. 18, no. 3, pp. 22-30, 2011.

[11] A. Damnjanovic, J. Montojo, J. Cho, H. Ji, J. Yang, and P. Zong, "UE's role in LTE advanced heterogeneous networks," IEEE Communications Magazine, vol. 50, no. 2, pp. 164-176, 2012.

[12] 3GPP TR 36.921, "FDD Home eNB (HeNB) Radio Frequency (RF) requirement analysis (Release 9)," March 2010.

[13] "Interference management in OFDMA Femtocells," Femto Forum whitepaper, March 2010.
[14] M. M. Wang and T. Ji, "Dynamic resource allocation for interference management in orthogonal frequency division multiple access cellular communications," IET Communications, vol. 4, no. 6, pp. 675-682, 2010.

[15] P. Wang, C. Liu, and R. Mathar, "Dynamic fractional frequency reused proportional fair in time and frequency scheduling in OFDMA networks," in Proceedings of 8th International Symposium on Wireless Communication Systems (ISWCS '11), pp. 745-749, November 2011.

[16] 3GPP TR 25.967, "Home Node B Radio Frequency (RF) Requirements (FDD) (Release 9)," May 2009.

[17] Z. Lu, Y. Sun, X. Wen, T. Su, and D. Ling, "An energyefficient power control algorithm in femtocell networks," in Proceedings of 7th International Conference on Computer Science \& Education (ICCSE '12), pp. 395-400, July 2012.

[18] A. Barbieri, A. Damnjanovic, T. Ji et al., "LTE femtocells: system design and performance analysis," IEEE Journal on Selected Areas in Communications, vol. 30, no. 3, pp. 586-594, 2012.

[19] M. K. Karakayali, G. J. Foschini, and R. A. Valenzuela, "Network coordination for spectrally efficient communications in cellular systems," IEEE Wireless Communications, vol. 13, no. 4, pp. 5661, 2006.

[20] R. Irmer, H. Droste, P. Marsch et al., "Coordinated multipoint: concepts, performance, and field trial results," IEEE Communications Magazine, vol. 49, no. 2, pp. 102-111, 2011.

[21] D. Lee, H. Seo, B. Clerckx et al., "Coordinated multipoint transmission and reception in LTE-advanced: deployment scenarios and operational challenges," IEEE Communications Magazine, vol. 50, no. 2, pp. 148-155, 2012.

[22] J. Lee, Y. Kim, H. Lee et al., "Coordinated multipoint transmission and reception in LTE-advanced systems," IEEE Communications Magazine, vol. 50, no. 11, pp. 44-50, 2012.

[23] K. S. Gomadam, V. R. Cadambe, and S. A. Jafar, "A distributed numerical approach to interference alignment and applications to wireless interference networks," IEEE Transactions on Information Theory, vol. 57, no. 6, pp. 3309-3322, 2011.

[24] A. Adhikary, V. Ntranos, and G. Caire, "Cognitive femtocells: breaking the spatial reuse barrier of cellular systems," in Proceedings of the Information Theory and Applications Workshop (ITA '11), pp. 56-65, February 2011.

[25] H. Dahrouj and W. Yu, "Multicell interference mitigation with joint beamforming and common message decoding," IEEE Transactions on Communications, vol. 59, no. 8, pp. 2264-2273, 2011.

[26] R1-084482, "Low-Complexity Precoding for LTE-A Collaborative MIMO: A Signal Leakage Approach," Mitsubishi Electric, 3GPP TSG RAN1 Meeting \#55, November 2008.

[27] R1-093411, "MU/CoMP Performance Comparison of Several Feedback Types," Motorola, 3GPP TSG RAN1 Meeting \#58, August 2009.

[28] R1-104661, “Comparison of Time-domain eICIC Solutions," LG Electronics, 3GPP TSG RAN1 Meeting \#62, August 2010.

[29] X. Shang, B. Chen, and M. J. Gans, "On the achievable sum rate for MIMO interference channels," IEEE Transactions on Information Theory, vol. 52, no. 9, pp. 4313-4320, 2006.

[30] M. Sadek, A. Tarighat, and A. H. Sayed, "A leakage-based precoding scheme for downlink multi-user MIMO channels," IEEE Transactions on Wireless Communications, vol. 6, no. 5, pp. 1711-1721, 2007.

[31] R3-111897, "X2 mobility between Macro and closed/hybrid HeNB,” CATT, 3GPP TSG RAN WG3 Meeting \#73, August 2011. 
[32] R3-112079, "Scenario for mobility enhancement between HeNB and eNB," Samsung, 3GPP TSG RAN WG3 Meeting \#73, August 2011.

[33] R3-112052, "Rel-11 HeNB Enhanced Mobility Scenarios," Ericsson, 3GPP TSG RAN WG3 Meeting \#73, August 2011.

[34] "Wireless in the home \& office: the need for both 3G Femtocells and Wi-Fi access points," Femto Forum, January 2010.

[35] "IEEE 802.16m Evaluation Methodology Document (EMD)," IEEE 802.16 Broadband Wireless Access Working Group. 

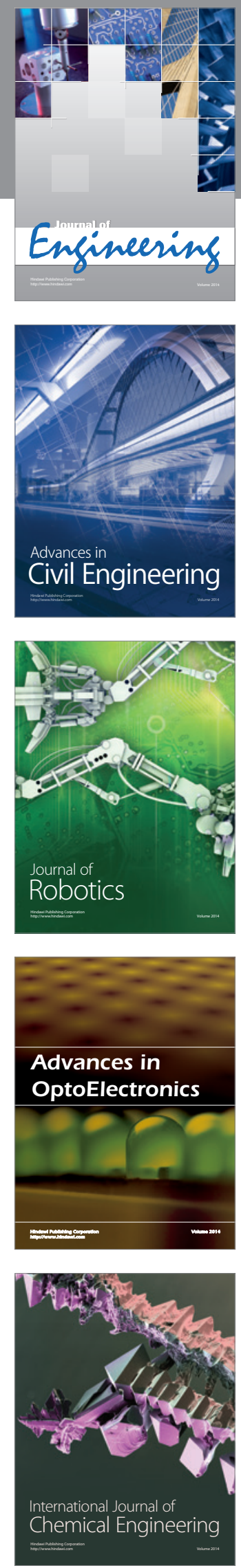

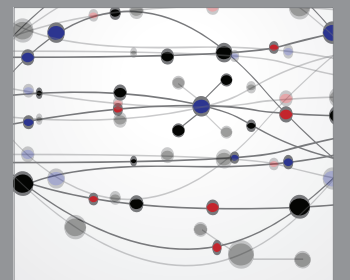

The Scientific World Journal
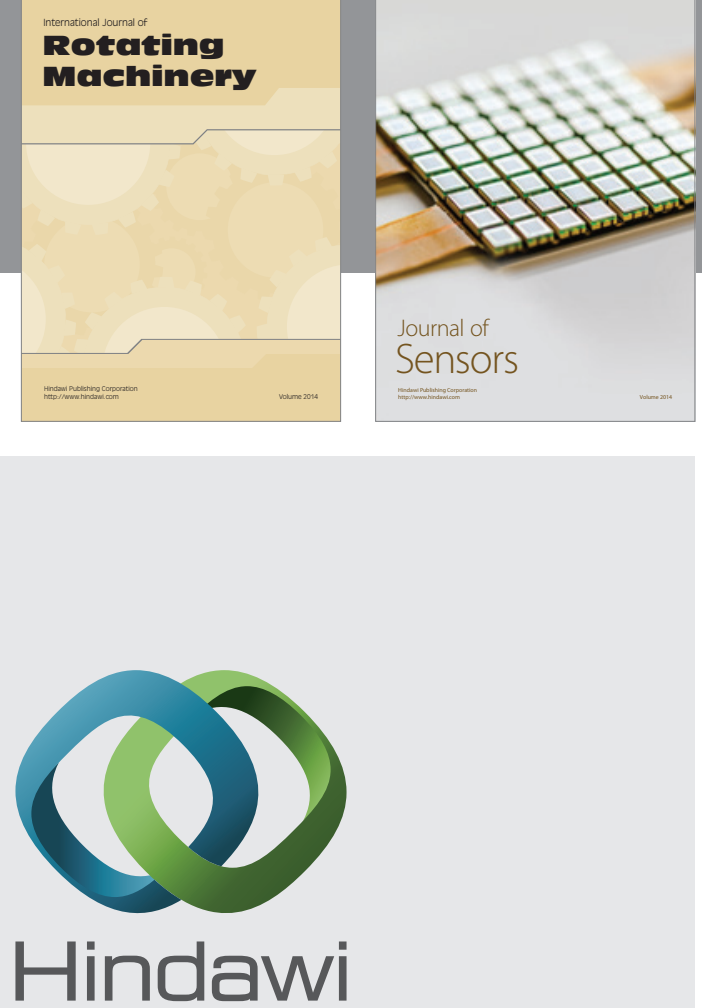

Submit your manuscripts at http://www.hindawi.com
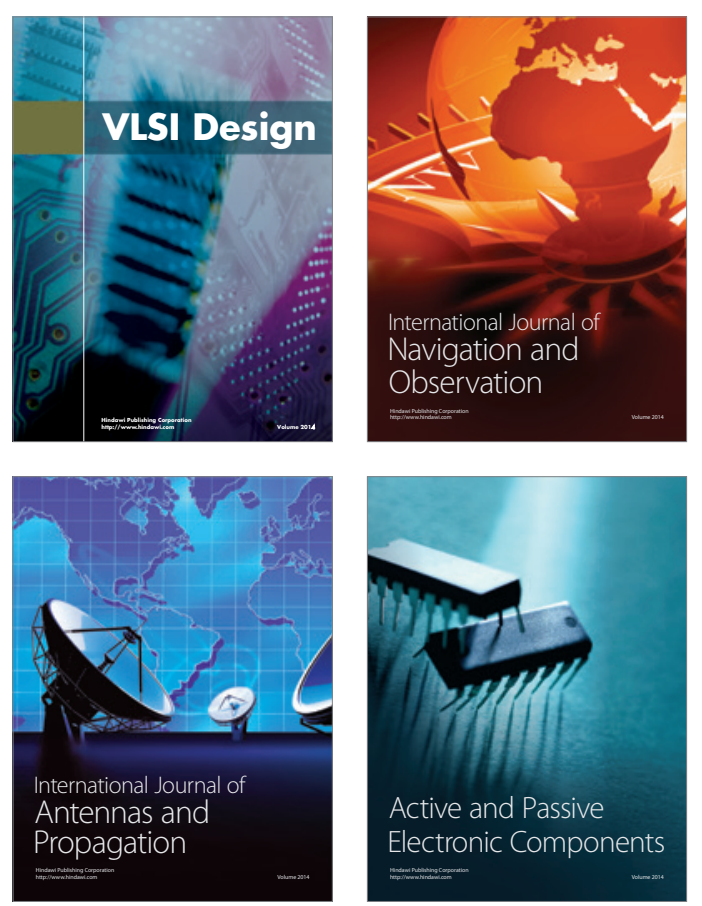
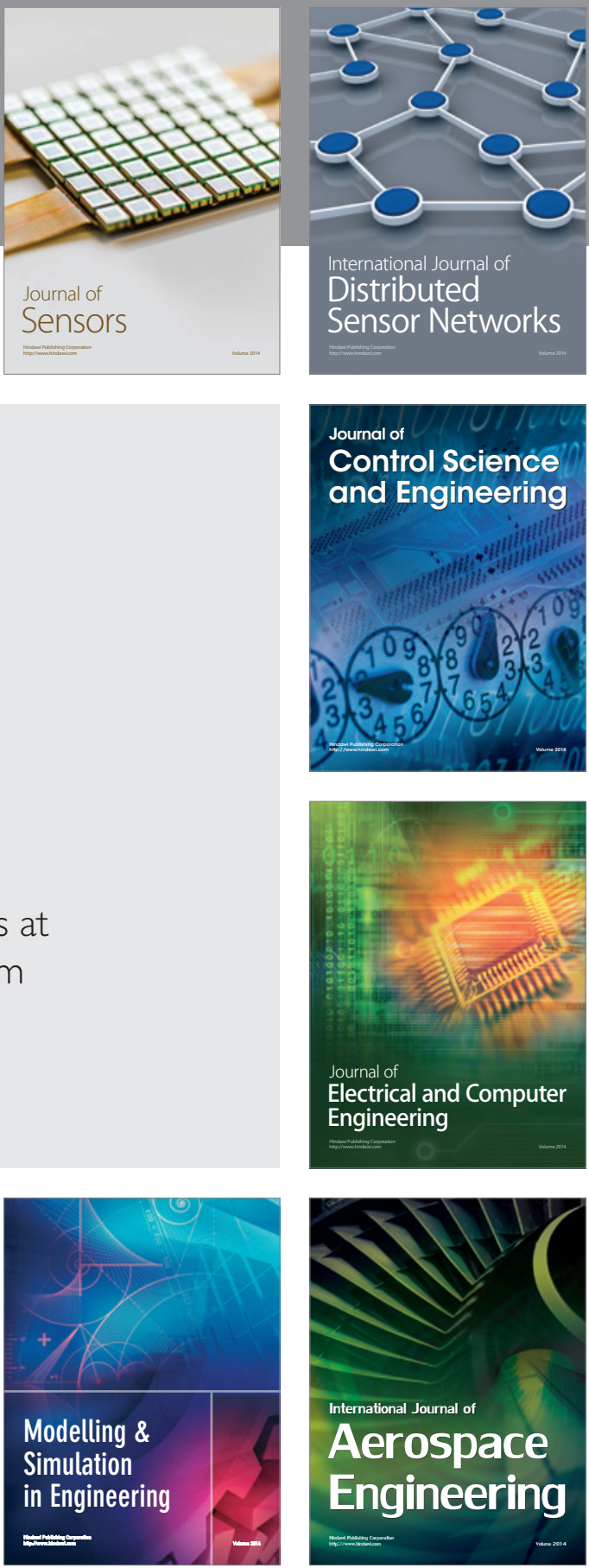

Journal of

Control Science

and Engineering
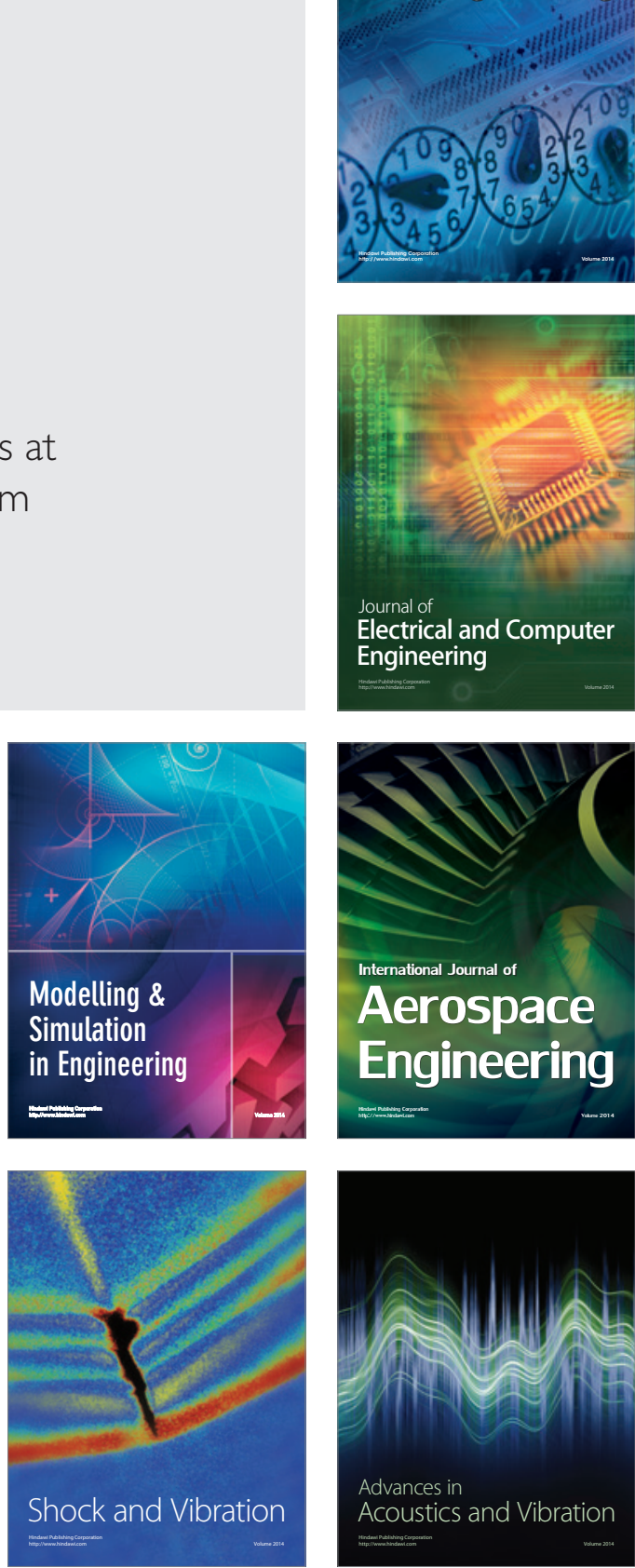